\title{
miR-144-3p and miR-425-5p are Negatively Associated With Atrial Fibrosis and Promote Atrial Remodeling by Targeting CREB1 in Atrial Fibrillation
}

\section{Feiyu Wei}

The first people's hospital of yunnan province

Li LV

the Second Affiliated Hospital of Kunming Medical University

\section{Xiaohui Kuang}

The First People's Hospital of Yunnan Province

\section{Wenjun Ren}

The First People's Hospital of Yunnan Province

Jie Fan ( $\square$ fanj913@sina.com )

The First People's Hospital of Yunnan Province

\section{Research}

Keywords: Atrial fibrillation, miRNAs, Atrial fibrosis, CREB1, Atrial remodeling

Posted Date: December 11th, 2020

DOl: https://doi.org/10.21203/rs.3.rs-125385/v1

License: (c) (i) This work is licensed under a Creative Commons Attribution 4.0 International License. Read Full License 


\section{Abstract}

Background: Progression of atrial fibrosis is vital for atrial remodeling in atrial fibrillation (AF). The main objective of this study was to explore the association between miR-144-3p and miR-425-5p, and atrial fibrosis as well as the resultant impact on atrial remodeling in AF.

Methods and Results: Through miRNAs sequencing and qRT-PCR, we demonstrated that miR-144-3p and miR-425-5p were downregulated in plasma and atrial tissue among the patients who suffered from AF. We confirmed that the plasma's miRNAs level was negatively correlated with left atrial fibrosis, which was evaluated with the left low voltage area using left atrial voltage matrix mapping. Catheter ablation restored decreased plasma miR-144-3p and miR-425-5p. Besides, ROC curve analysis revealed that the miRNAs not only differentiated AF from healthy control of AUC 0.928 and 0.921 , respectively, but also discriminated persistent AF from paroxysmal AF of AUC 0.906 and 0.888 , respectively. Furthermore, the downregulated miR-144-3p and miR-425-5p promoted atrial fibroblast proliferation by CCK-8. CREB1 was realized to be a common direct target for miR-144-3p and miR-425-5p by western blot and luciferase assay.

Conclusions: Our findings suggested that miR-144-3p and miR-425-5p could serve as novel atrial fibrosis biomarkers and hence contribute to atrial remodeling in AF.

\section{Introduction}

Atrial fibrillation (AF) is a common atrial arrhythmia, which increases the risk of stroke, congestive heart failure and myocardial infarction ${ }^{[1,2]}$. Radiofrequency catheter ablation has emerged as an important treatment option with favorable outcomes in patient with symptomatic, drug-resistant paroxysmal AF. Nevertheless, long-term recurrence is still prevalent in persistent $\mathrm{AF}^{[3,4]}$.

Atrial structural remodeling is the main pathological mechanism for the development of AF. Atrial fibrosis, which is induced by the proliferation of atrial fibroblast, myocytes apoptosis, and excessive deposition of intercellular collagen, is the most prominent pathological manifestation of structural remodeling ${ }^{[5]}$. Previous studies have shown that the severity of atrial fibrosis is related to the prognosis of AF. Homogenized left atrial fibrosis matrix can reduce the recurrence rate ${ }^{[6]}$. Therefore, seeking early biomarkers of atrial fibrosis and exploring the mechanism of structural remodeling have become necessary in the field of AF.

The microRNA(miRNA) is a short non-coding RNA molecule with about 22 nucleotides. It regulates gene silencing by degrading target mRNA or inhibiting protein translation through complementary combining the $3^{\prime}$-untranslated regions (UTRs) of $m R N A^{[7]}$. Previous study demonstrated that miRNAs are widely involved in regulating cardiovascular functions and pathogenesis ${ }^{[8,9]}$. Multiple studies have suggested that circulating miRNAs could be used as diagnostic biomarkers in various diseases ${ }^{[10,11]}$. Circulating miRNAs were also closely related to the procedure outcome in patients undergoing radiofrequency 
catheter ablation ${ }^{[12]}$, and radiofrequency catheter ablation can restore plasma altered miRNAs ${ }^{[13]}$. Recently, several studies have shown that miRNA also contributed to the regulation of atrial remodeling in $\mathrm{AF}^{[14,15]}$.

The present study was aimed to screen the fibrosis biomarkers for AF and investigate its role in atrial structure remodeling. We found that miR-144-3p and miR-425-5p were downregulated in the plasma and atrial tissue in AF unlike in the healthy control. They were also negatively correlated with the left atrial fibrosis in persistent AF. Besides, it was discovered that catheter ablation could restore decreased plasma miR-144-3p and miR-425-5p. Downregulation of miR-144-3p and miR-425-5p could promote atrial fibroblast proliferation and subsequently result into fibrosis by targeting CREB1. These results suggest these miRNAs could be potential as non-invasive biomarkers for atrial fibrosis in AF.

\section{Materials And Methods}

\subsection{Study population}

This study included three cohorts. The first was the miRNAs sequencing cohort which included 3 patients suffering from $A F$ and 3 healthy controls. The second was the validation cohort which consisted a total of $80 \mathrm{AF}$ (40 persistent AF and 40 paroxysmal AF) who were hospitalized in our department from October 2018 to October 2019. 80 genderand age-matched individuals without AF were enrolled as the control. 60 of them received radiofrequency catheter ablation. The third cohort included 6 valvular AF who underwent cardiac surgical operation, and 6 non-AF who undergoing cardiac surgical operation as the controls. Left atrial diameter, left ventricular ejection fraction and left ventricular end-diastolic diameter were assessed by transthoracic echocardiographic.

The study exclusion criteria of the first and second cohort were age $<18$ years or $>80$ years, severe underlying structural heart disease, pregnancy, contraindication for anticoagulation, serious liver or renal dysfunction, LA diameter $\geq 50 \mathrm{~mm}$, and LA or LAA thrombosis. This study was approved by the Ethics Committees of the First People's Hospital of Yunnan Province. All the participants signed a written informed consent document. All of the procedures were done following the declamiceion of Helsinki and relevant policies in China.

\subsection{Plasma and atrial tissue collection}

Blood samples were collected in the morning with anti-EDTA tubes and processed within $1 \mathrm{~h}$. They were subjected to centrifugation at $2000 \mathrm{~g}$ for $10 \mathrm{~min}$, and then stored at $-80^{\circ} \mathrm{C}$ until further analysis. Rightatrial tissue specimens were collected after the right atrium was opened and preserved in liquid nitrogen for subsequent experiments.

\section{3 qRT-PCR}


A neasy mini kit (Qiagen, Germany) was used to isolate the total RNAs according to the manufacturer's instruction. Briefly, $1 \mu \mathrm{g}$ total RNA was reverse transcribed with Anchored-oligo (dT) 18 Primer for cDNA synthesis using Transcriptor First Strand cDNA Synthesis Kit (Roche, Switzerland) based the manufacturer's instruction. The polymerase chain reaction was performed in a $10 \mu$ l reaction mixture on an ABI 7900 real-time PCR instrument (Applied Biosystems, USA). The mixture was heated for 1 minute at $95^{\circ} \mathrm{C}$ for predenatumiceion, and then processed to 35-40 PCR cycles, which consisted denatumiceion at $95^{\circ} \mathrm{C}$ for 15 seconds, annealing at $60^{\circ} \mathrm{C}$ for 60 seconds, and extension at $95^{\circ} \mathrm{C}$ for 15 seconds. The miR- 16 served as an internal reference of plasma miRNAs. U6 and GAPDH were used as an internal reference of miRNA and mRNA for tissue and cells. $2^{-\Delta \Delta C t}$ method was used to calculate the relative expression level. The changes in miRNAs expression were calculated using $\Delta \mathrm{Ct}$, whereby a lower $\Delta \mathrm{Ct}$ value meant a higher expression of miRNA.

\section{4 miRNA Solexa sequencing}

Solexa sequencing was carried out to analyze plasma miRNA expression from the first cohort subjects. Through illumina sequencing, miRNA digitalization analysis was performed based on Hiseq4000 highthroughput sequencing. All the procedures were performed with respect to the manufacturer's guidelines. At least 30 copies in either group and at least a two-fold difference in expression between the two groups was used to screen for miRNA. Differentially expressed miRNAs were defined as the fold changes of 2 or higher and P-values of 0.05 or lower relative to the controls.

\subsection{Left atrial voltage matrix mapping}

Atrial fibrillation with indication for radiofrequency catheter ablation was enrolled after preoperative preparation. Local anesthesia and conscious sedation with fentanyl were used on all the study subjects. Two long sheaths (Swartz, SL1, 8.5F, St. Jude Medical, Minnesota, USA) were inserted into the femoral vein. Puncture of the atrial septum was performed twice. All the patients were subjected to the circumferential pulmonary vein isolation $(\mathrm{PVI})$. Left atrial voltage matrix mapping was started in case the atrial fibrillation converted to sinus rhythm post-PVI, or the electrical cardioversion was performed before left atrial voltage matrix mapping. Left atrial voltage matrix mapping was performed under sinus rhythm with a pentary mapping catheter (pentary catheter, Biosense Webster, Diamond Bar, CA). It was created with a 3-dimensional mapping system (Carto-3, Biosense Webster), with the acquisition of 300 to 1000 points. The range of mapping voltage amplitude was set at 0.1-0.4 mV. The local electrogram that was lower than $0.4 \mathrm{mV}$, was considered as a low voltage area (LVA), and it represented the extent of left atrial fibrosis $^{[16]}$. The extent of left atrial LVA was calculated as a percentage of the LVA to the left atrial surface area.

\subsection{Isolation of atrial fibroblast}

Using the classic surgery procedures, the mouse's heart was removed. The excess tissue was cut off. The atrial tissue was minced with scissors and digested at $37^{\circ} \mathrm{C}$ in phosphate-buffered saline (PBS) that contained $0.1 \%$ trypsin (Gibco, BRL) and 0.05\% type I collagenase (Gibco, BRL). After 8-10 cycles of 
digestion, for $6 \mathrm{~min}$ each, the digestive solution was centrifuged at low-speed centrifugation for 5 minutes. The supernatant was discarded and the cells were suspended in DMEM (Gibco, BRL) containing 15\% fetal bovine serum (Gibco, BRL). Percoll fluid (Sigma, USA) was used to purify the cardiomyocytes and fibroblasts. Then the fibroblasts were appropriately diluted and cultured adherently for further analysis.

\subsection{CCK-8}

Cell counting kit-8 (CCK-8) was used to detect fibroblasts proliferation. The fibroblasts were seeded in 96well plates at a density of $2 \times 10^{3} /$ well. After transfection with miRNA for 48 hours, $10 \mu$ l CCK- 8 solution was added to a $90 \mu \mathrm{l}$ culture medium, and then incubated at $37^{\circ} \mathrm{C}$ in $5 \% \mathrm{CO} 2$ for 2 hours. The optical density (OD) value was recorded on the $450 \mathrm{~nm}$ wavelength enzyme-label analyzer.

\subsection{Luciferase report assay}

Luciferase reporter assay was described in detail in our previous publication ${ }^{[17]}$. Briefly, DNA segments containing 3'UTRs of human CREB1 mRNA were inserted into EcoRI and Xhol sites of pcDNA3.0-EGFP (TaKaRa, China). HL-1 cells were cotransfected with 1.0 $\mathrm{\mu g}$ firefly luciferase reporter vectors containing the target sites, $100 \mathrm{nM}$ miR-144-3p, or miR-425-5p mimics or miR-control and $0.06 \mu \mathrm{g}$ Renilla luciferase control vectors using Lipofectamine3000 (Invitrogen, USA). The cells were harvested after 48 hours. The results were expressed as relative luciferase activity (firefly luciferase/Renilla luciferase). The mutation of the miRNAs binding sites for human CREB1 3'UTRs was generated using the Quick Change XL SiteDirected Mutagenesis Kit.

\subsection{Western blot}

Total protein extracts were obtained via lysis buffer containing protease inhibitor cocktail (Sigma). The proteins were transferred to polyvinylidene difluoride membranes and blocked in PBS containing a $5 \%$ fatfree milk powder. Then the antibodies against CREB1 (Abcam, UK) were used to detect the corresponding proteins. Goat anti-rabbit immunoglobulin conjugated to horseradish peroxidase (CST, USA) was used as the secondary antibody. It was scanned and photographed in the BioRAD gel imager. The relative expression of CREB1 was determined using the specific bands densities in western blots, normalized to the density of GAPDH.

\subsection{Statistical analysis}

Data were presented as mean $\pm S D$ or median [interquartile range] if their values were not normally distributed. Unpaired Student's t-tests or non-parametric Mann-Whitney U-test was used to compare the continuous variables. Categorical variables were presented as absolute or relative frequencies, and compared using the $\chi 2$ test. The correlation between miRNA and the relative parameter were evaluated using the Pearson's correlation coefficients. The valve was log-transformed to normalize their distribution before statistical analysis. Moreover, receiveroperatingcharacteristic (ROC) curves analysis was performed and the area under the curves (AUC) was calculated to evaluate the candidate miRNAs' 
diagnosis power. P-value of $<0.05$ was considered statistically significant. SPSS 17.0 and GraphPad Prism7.0 software was used for all the statistical analyses and graph presentation.

\section{Results}

\subsection{Baseline characteristics of the subjects}

The baseline demographic characteristics of the three cohorts were shown in Online Table1 to Table3. The distributed characteristics were similar between the two groups. 30 paroxysmal AF and 30 persistent $\mathrm{AF}$ received radiofrequency catheter ablation in the second cohort.

\subsection{Plasma miRNAs level in atrial fibrillation}

The top 25 downregulated and upregulated differences in miRNAs expression between the two groups were illustrated with a heatmap (Figure 1A). The screening criteria indicated that, 4 miRNAs (hsa-miR-302, hsa-miR-193a-5p, hsa-miR-153-3p, hsa-miR-551-3p) were upregulated in the AF group, while 6 miRNAs (hsa-miR-199-3p, hsa-miR-1260, hsa-miR-144-3p, hsa-miR-425-5p, hsa-miR-21, hsa-miR-22-3p) were downregulated (Table 1).

\subsection{Comparing the qRT-PCR and miRNAs sequencing}

The miR-199-3p and miR-22-3p were studied in our previous study ${ }^{[18]}$. And miR-21 were demonstrated positively associated with atrial fibrosis in AF in other study ${ }^{[12]}$. The results demonstrated that hsa-miR302, hsa-miR-193a-5p, hsa-miR-153-3p, and hsa-miR-551-3p were upregulated, whereas hsa-miR-1260, hsa-miR-144-3p, hsa-miR-425-5p were downregulated. These results were similar to the miRNA sequencing results (Figure1B-C).

\subsection{Verification of miRNAs}

7 candidate miRNAs were detected in an independent cohort of $80 \mathrm{AF}$ patients and 80 healthy controls by qRT-PCR. The miR-144-3p and miR-425-5p were downregulated in the AF group whereas hsa-miR-302 was up-regulated. This was consistent with the results in the first miRNA sequencing cohort. The expression of the other 4 miRNAs was inconsistent with the sequencing results (Figure 2A-C). We found that the expression of miR-144-3p and miR-425-5p was downregulated in left atrial blood than in the peripheral blood. On the contrary, expression of miR-302 was upregulated in the left atrial blood than in the peripheral blood (Online Figure 1).

\section{5 miRNAs expression in atrial tissue in AF patients and mice AF model}

We established that the expression of miR-144-3p and miR-425-5p was decreased in the AF than in the SR whereas miR-302 was increased (Figure 2D-E). This was in agreement with the plasma results. We also realized that the expression of miR-144-3p and miR-425-5p was lower in the left atrial tissue compared to the right atrial tissue, and miR-302 expression was increased in the left atrium tissue in 
mouse AF model (Online Figure 2). Therefore, these findings implied that the expression level of miR-144$3 p$ and miR-425-5p was lowered, while that of miR-302 was increased in left atrial in comparison to the right atrial tissue in $\mathrm{AF}$.

\section{6 miRNAs expression in different type of AF}

To detect whether the differentially expressed miRNA was associated with AF type, the miR-302, miR-144$3 p$, and miR-425-5p were detected in 40 paroxysmal $A F$ and 40 persistent $A F$, and results showed the expression level of miR-144-3p and miR-425-5p was lower in persistent AF than in paroxysmal AF (Figures 2F-G). However, miR-302 expression difference was not significantly between the two different types of AF (Figure $2 \mathrm{H}$ ).

\subsection{Diagnostic value of the miRNAs for AF}

ROC analyses were performed on the AF and healthy individuals. All the three miRNAs could differentiate AF from healthy control with AUC of 0.928 (95\% Cl: $0.885-0.970,88 \%$ sensitivity and $89 \%$ specificity), for miR-144-3p, 0.921 (95\% Cl: 0.881-0.961, 95\% sensitivity and 77\% specificity) for miR-425-5p, 0.891 (95\% Cl: $0.838-0.945,86 \%$ sensitivity and $81 \%$ specificity) for miR-302, respectively (Fig3A). Considering the difference expression of miRNAs in paroxysmal and persistent AF, ROC curve analysis was carried out in paroxysmal and persistent AF, and the AUC was 0.906 (95\% Cl: 0.842-0.969, 73\% sensitivity and 99\% specificity ) of miR-144-3p, 0.888 (95\% Cl: 0.817-0.958, 85\% sensitivity and $85 \%$ specificity ) of miR-425$5 p$ and 0.681 (95\% Cl: 0.564-0.798, 45\% sensitivity and 83\% specificity ) of miR-302 (Fig 3B).

\subsection{Plasma level of miR-144-3p and miR-425-5p was negatively correlated with the left atrial fibrosis in persistent AF}

The left atrial fibrosis was evaluated using the extent of left atrial low voltage area (LVA) by left atrial voltage matrix mapping during radiofrequency catheter ablation in the persistent AF. The plasma miR144-3p and miR-425-5p level were negatively associated with the left atrial fibrosis by Pearson's correlation coefficients (Fig4A-B). However, the plasma miR-302 level was not found to be linked to the left atrial fibrosis (Fig4C).

We divided the left atrial low voltage area into 3 groups according to the extent of left atrial fibrosis (group 1: LVA extent between $0 \%$ to $10 \%$, group 2: LVA extent between $10 \%$ to $20 \%$, group 3: LVA extent between over 20\%), and the results showed that the expression levels of miR-144-3p and miR-425-5p were lowest in group 3 and the highest in the group1 (Online Figure 3 ).

\subsection{Comparison of plasma miRNA expression level in the pre-RFCA and post-RFCA}

The plasma miR-144-3p and miR-425-5p level were detected in 20 AF patients during pre-RFCA (radiofrequency catheter ablation) and post-RFCA, and also compared with the control group. The relative plasma level of miR-144-3p increased in post-RFCA compared with pre-RFCA (the median and interquartile ranges of pre-RFCA vs. post-RFCA were 1.10:0.34 and 0.762:0.2963, respectively). The level of 
miR-425-5p also increased (the median and inter-quartile ranges of pre-RFCA vs. post-RFCA were 1.03:0.60 and 0.49:0.28, respectively). Besides, the plasma miR-144-3p and miR-425-5p expression level were not significantly different between post-RFCA and the control group ( $p=0.108$ and 0.628 , respectively) (Figure 4D-F).

\subsection{Effect of miR-144-3p and miR-425-5p on atrial fibroblast proliferation and fibrosis}

Atrial fibroblast proliferation and fibrosis were the hallmark of atrial remodeling in AF. We investigated whether the aberrant expression of the miRNAs influence fibroblast proliferation and fibrosis. The miR144-3p and miR-425-5p inhibitor was transfected into the atrial fibroblast cell to downregulate the expression of miR-144-3p and miR-425-5p (Figure 5A). We found downregulation of miR-144-3p and miR425-5p promoted the atrial fibroblast proliferation (Figure 5B-C), and the proliferation marker expression of Ki67 and Cyclin D (Figure 5D). Besides, it also promoted the fibrosis marker expression of collagen I and collagen III (Figures 5E-F).

\subsection{CREB1 was the common direct target of miR-144-3p and miR-425-5p}

Two widely available mammalian target prediction programs (miRDB and TargetScan7.2 databases) were used to predict the target gene of the miRNAs. 645 and 97 targets of miR-144-3p and miR-425-5p respectively were indicated by miRDB and TargetScan7.2. 20 of them were among the common targets for miR-144-3p and miR-425-5p (Online Figure 4), including DIP2C, MAPK6, CPEB2, ZNF148, ATP1B1, CREB1, LCOR, SYNCRIP, CPEB1, IFFO2, SCAMP1, TM9SF3, AFF4, FST, ZFHX3, CREBZF, LARP4B, FBN1, GOLGA4, SMAD5. We then after analyzed the expression level of 20 genes in the atrial tissue in SR and AF (Primer showed in the Online Table4). We found only the expression of CREB1 was increased. Besides, FBN1 and ZFHX3 expression was decreased in AF than in SR (Figure 6A). The expression of miRNA was negatively correlated with the target gene expression, on this basis, we speculated that CREB1 may be the target gene for miR-144-3p and miR-425-5p. More importantly, our results also showed the expression of miR-144-3p and miR-425-5p was negatively associated with CREB1 expression in the atrial tissue in AF (Figures 6B-C). Finally, we found that inhibition of CREB1 expression could reverse the phenotype of down-regulation of miR-144-3p and miR-425-5p(Figure 6D-F).

The potential binding target of miR-144-3p and miR-425-5p with CREB1 3'-URT was predicted by TargetScan 7.2 (Figure 7A), and it was realized that the downregulation of miR-144-3p and miR-425-5p significantly promoted CREB1 protein expression in the fibroblast cells (Figure 7B-C). Furthermore, through luciferase assay, CREB1 was verified as the common direct target for miR-144-3p and miR-425$5 p$ (Figure7D-E).

\section{Discussion}

This study demonstrated that miR-144-3p and miR-425-5p were downregulated in the plasma as well as in atrial tissue in AF. And downregulation of miR-144-3p and miR-425-5p were negatively correlated with left atrial fibrosis and cntribured to atrial remodeling by targeting CREB1. Besides, catheter ablation could 
restore the decreased miR-144-3p and miR-425-5p in the plasma. This findings suggested that miR-144$3 p$ and miR-425-5p could serve as noninvasive early diagnostic biomarkers for AF as well as a predictive biomarkers for atrial fibrosis in AF.

Diagnosis of $A F$ is dependent on the clinical feature and ECG monitoring characterized by disappeared $P$ wave and irregular R-R interval ${ }^{[19]}$. However, a considerable proportion of AF is asymptomatic ${ }^{[20]}$. Hence, a sensitive and specific non-invasive diagnostic technique should be sought to identify the asymptomatic atrial fibrillation during the early stage. Accumulating pieces of evidence suggest that miRNAs are pivotal regulators for cardiovascular functions. The circulating miRNAs could serve as a non-invasive diagnostic biomarker since they carry disease-specific information ${ }^{[17,21]}$. Meanwhile, the miRNAs can severe as prognosis biomarker of various cardiovascular diseases due to the alter dynamically in different stages of disease ${ }^{[22]}$. In this study, we found that miR-144-3p and miR-425-5p were downregulated in plasma in AF patients. The expression of miR-144-3p and miR-425-5p was also lower in persistent atrial fibrillation than in paroxysmal atrial fibrillation. These results suggest that the miRNAs experience changes during different stages of AF. Besides, ROC curve analysis showed a lower level of miR-144-3p and miR-425-5p and higher level of miR-302 could not only differentiate AF from a healthy individual but also differentiate persistent AF from paroxysmal AF. The implication is that miR-144-3p and miR-425-5p could serve as a potential diagnostic biomarkers for AF.

Atrial fibrosis is an important hallmark for atrial structural remodeling, and hence it forms an important substrate for $\mathrm{AF}^{[5]}$, The extent of left atrial fibrosis was more serious in the persistent $A F$ than paroxysmal AF, which was positively associated with AF prognosis ${ }^{[23,24]}$. Pulmonary vein isolation and scar homogenization were also associated with considerably better long-term outcomes than the pulmonary vein isolation alone in $\mathrm{AF}$ with severe left atrial scars ${ }^{[25]}$. On this basis, an early recognition and intervention of atrial fibrosis was the effective method to improve the prognosis of AF. The cardiac $\mathrm{MRI}$ and intraoperative left atrial voltage mapping are the main methods for evaluating atrial fibrosis extent ${ }^{[26]}$. However, these methods are either invasive or complex. As such, seeking noninvasive sensitivity fibrosis biomarkers, which can not only evaluate atrial fibrosis but also provide evidence for the selection of the appropriate procedures becomes apparent, so as to improve the prognosis. Our study established that the plasma miR-144-3p and miR-425-5p were negatively correlated with the left atrial fibrosis in persistent AF. We also discovered that catheter ablation could restore the decreased miR-144$3 p$ and miR-425-5p. These results suggested that miR-144-3p and miR-425-5p may serve as sensitive prediction biomarkers for atrial fibrosis, thereby providing clues for assessing the pathogenic contribution of the miRNAs in AF. Furthermore, we found the expression of miR-144-3p and miR-425-5p was lower in the left atrial blood than in the peripheral blood. This implied that, the aberrant expression of miR-144-3p and miR-425-5p in the left atrial blood play a critical role in atrial fibrillation.

Atrial remodeling is the main pathological mechanism for the occurrence and persistence of $\mathrm{AF}^{[27]}$. Atrial fibrosis induced by the proliferation of atrial fibroblasts, cardiomyocytes apoptosis, and over deposition of the intercellular matrix, was the prominent pathological manifestation in structural remodeling ${ }^{[15]}$. 
Atrial electrical remodeling characterized by the shortening of the effective refractory period and action potential duration, is another fundamental trigger for atrial fibrillation ${ }^{[28]}$. However, the prevention and treatment of atrial fibrillation are mainly focused on the improvement of atrial electrical remodeling. For instance, the antiarrhythmic drugs can only recover and prolong the effective refractory period of the atrium ${ }^{[29]}$. Radiofrequency catheter ablation normally focuses on the removal of the abnormal local excitations and blocks the conduction between the pulmonary vein and the left atrium. As such, it becomes necessary to explore the molecular mechanism of structural remodeling. Our results implied that the downregulation of miR-144-3p and miR-425-5p could promote fibroblast cell proliferation and hence cause fibrosis by targeting CREB1. This suggested that the inappropriate down-regulation of miR144-3p and miR-425-5p in the atrial tissue and plasma could facilitate the occurrence and development of atrial fibrillation via atrial remodeling.

Previous studies have reported the correlation between miRNAs and atrial fibrillation. For example, The expression of miR-30d was upregulated in patients with persistent $A F$, and upregulation of miR-30d promoted atrial electrical remodeling by targeting $\mathrm{KCNJ}^{[30]}$. Besides, miR-133 and miR-590 were downregulated in canine $A F$ model and contributed to $A F$ development via regulating structure remodeling ${ }^{[31]}$. However, the relationship between miRNA and AF need to be further clarified. In this study, we found two novelty miRNAs, which were associated with atrial fibrosis and contributed atrial structure remodeling.

\section{Conclusions}

The present study demonstrated that the expression levels of miR-144-3p and miR-425-5p decreased in the plasma in AF. However, catheter ablation could restore the declined miR-144-3p and miR-425-5p levels. Besides, miR-144-3p and miR-425-5p could serve as non-invasive biomarkers for atrial fibrosis and consequently regulate atrial remodeling by targeting CREB1.

\section{Limitation}

The study had two main limitations as follows: Firstly, the relationship between the candidate miRNAs and the recurrence of AF after radiofrequency catheter ablation was not assessed. Secondly, only the left atrial voltage matrix mapping was used to evaluate atrial fib rosis, other methods such as cardiac MRI were not utilized ${ }^{[32]}$.

\section{Abbreviations}

AF: Atrial fibrillation

ROC: receiveroperatingcharacteristic

\section{Declarations}


Ethics approval and consent to participate: This study was approved by the Ethics Committees of the First People's Hospital of Yunnan Province. All the participants signed a written informed consent document. All of the procedures were done following the declamiceion of Helsinki and relevant policies in China.

Consent for publication: Consent for publication of all the data.

Availability of data and materials: The datasets used and/or analysed during the current study are available from the corresponding author on reasonable request.

Competing interests: The authors declare that they have no competing interests

Funding: Financial support was provided by the Project of Yunnan Arrhythmia Research Center [Grant Number: 2018NS0267 and 2018NS0268]. And Department of science and Technology of Yunnan Province-Kunming Medical University applied basic research [Grant Number:2019FE001(-291).

Authors' contributions: Feiyu Wei did the clinical sample experiment and analyzed data, and was a major contributor in writing the manuscript. Li LV was responsible for cell experiment and analyzed data. Xiaohui Kuang was responsible for Left atrial voltage matrix mapping. Wenjun Ren was responsible for sample collection. Jie Fan was responsible for the design and revising manuscripts.All authors read and approved the final manuscript.

Acknowledgements:The authors acknowledge the contributions of the participating patients and healthy controls.

\section{References}

1. Odutayo, A., C.X. Wong, A.J. Hsiao, S. Hopewell, D.G. Altman and C.A. Emdin, Atrial fibrillation and risks of cardiovascular disease, renal disease, and death: systematic review and meta-analysis. BMJ, 2016. 354: p. i4482.

2. Bekwelem, W., S.J. Connolly, J.L. Halperin, S. Adabag, S. Duval, S. Chrolavicius, et al., Extracranial Systemic Embolic Events in Patients With Nonvalvular Atrial Fibrillation. Circulation, 2015. 132(9): p. 796-803.

3. Tilz, R.R., C.H. Heeger, A. Wick, A.M. Saguner, A. Metzner, A. Rillig, et al., Ten-Year Clinical Outcome After Circumferential Pulmonary Vein Isolation Utilizing the Hamburg Approach in Patients With Symptomatic Drug-Refractory Paroxysmal Atrial Fibrillation. Circ Arrhythm Electrophysiol, 2018. 11(2): p. e005250.

4. Tilz, R.R., A. Rillig, A.M. Thum, A. Arya, P. Wohlmuth, A. Metzner, et al., Catheter ablation of longstanding persistent atrial fibrillation: 5-year outcomes of the Hamburg Sequential Ablation Strategy. J Am Coll Cardiol, 2012. 60(19): p. 1921-1929. 
5. Thanigaimani, S., D.H. Lau, T. Agbaedeng, A.D. Elliott, R. Mahajan and P. Sanders, Molecular mechanisms of atrial fibrosis: implications for the clinic. Expert Rev Cardiovasc Ther, 2017. 15(4): $p$. 247-256.

6. Yang, B., C. Jiang, Y. Lin, G. Yang, H. Chu, H. Cai, et al., STABLE-SR (Electrophysiological Substrate Ablation in the Left Atrium During Sinus Rhythm) for the Treatment of Nonparoxysmal Atrial Fibrillation: A Prospective, Multicenter Randomized Clinical Trial. Circ Arrhythm Electrophysiol, 2017. 10(11).

7. Stark, A., J. Brennecke, N. Bushati, R.B. Russell and S.M. Cohen, Animal MicroRNAs confer robustness to gene expression and have a significant impact on 3'UTR evolution. Cell, 2005. 123(6): p. 1133-1146.

8. Wojciechowska, A., A. Braniewska and K. Kozar-Kaminska, MicroRNA in cardiovascular biology and disease. Adv Clin Exp Med, 2017. 26(5): p. 865-874.

9. Divakaran, V. and D.L. Mann, The emerging role of microRNAs in cardiac remodeling and heart failure. Circ Res, 2008. 103(10): p. 1072-1083.

10. Fan, P.C., C.C. Chen, C.C. Peng, C.H. Chang, C.H. Yang, C. Yang, et al., A circulating miRNA signature for early diagnosis of acute kidney injury following acute myocardial infarction. J Transl Med, 2019. 17(1): p. 139.

11. Aghabozorgi, A.S., N. Ahangari, T.E. Eftekhaari, P.N. Torbati, A. Bahiraee, R. Ebrahimi, et al., Circulating exosomal miRNAs in cardiovascular disease? Pathogenesis: New emerging hopes. J Cell Physiol, 2019.

12. Zhou, Q., C. Maleck, S.N.I. von Ungern-Sternberg, B. Neupane, D. Heinzmann, J. Marquardt, et al., Circulating MicroRNA-21 Correlates With Left Atrial Low-Voltage Areas and Is Associated With Procedure Outcome in Patients Undergoing Atrial Fibrillation Ablation. Circ Arrhythm Electrophysiol, 2018. 11(6): p. e006242.

13. Liu, T., S. Zhong, F. Rao, Y. Xue, Z. Qi and S. Wu, Catheter ablation restores decreased plasma miR409-3p and miR-432 in atrial fibrillation patients. Europace, 2016. 18(1): p. 92-99.

14. Galenko, O., V. Jacobs, S. Knight, M. Taylor, M.J. Cutler, J.B. Muhlestein, et al., The role of microRNAs in the development, regulation, and treatment of atrial fibrillation. J Interv Card Electrophysiol, 2019.

15. Nattel, S., Molecular and Cellular Mechanisms of Atrial Fibrosis in Atrial Fibrillation. JACC Clin Electrophysiol, 2017. 3(5): p. 425-435.

16. Herczeg, S., K. Walsh, J.J. Keaney, E. Keelan, J. Travers, G. Szeplaki, et al., Quantitative assessment of left atrial scar using high-density voltage mapping and a novel automated voltage analysis tool. $J$ Interv Card Electrophysiol, 2019.

17. Zhang, J., F. Wei, L. Ding, L. Wang, X. Zhang, L. Yu, et al., MicroRNA-1976 regulates degeneration of the sinoatrial node by targeting Cav1.2 and Cav1.3 ion channels. J Mol Cell Cardiol, 2019. 134: p. 7485.

18. Wei FY, Fan J, Gao T, Wang LL. The effect of miR-199-3p on fibroblasts proliferation to promote atrial structural remodeling by targeting SP1 in atrial fibrillation. 
19. Journal of Clinical Cardiovascular Disease. 2018,36(6). 523-530.19. January, C.T., L.S. Wann, H. Calkins, L.Y. Chen, J.E. Cigarroa, J.C. Cleveland, Jr., et al., 2019 AHA/ACC/HRS Focused Update of the 2014 AHA/ACC/HRS Guideline for the Management of Patients With Atrial Fibrillation: A Report of the American College of Cardiology/American Heart Association Task Force on Clinical Practice Guidelines and the Heart Rhythm Society in Collaboration With the Society of Thoracic Surgeons. Circulation, 2019. 140(2): p. e125-e151.

20. O'Neal, W.T., T. Salahuddin, S.T. Broughton and E.Z. Soliman, Atrial Fibrillation and Cardiovascular Outcomes in the Elderly. Pacing Clin Electrophysiol, 2016. 39(9): p. 907-913.

21. E, S., M.C. Costa, S. Kurc, A. Drozdz, N. Cortez-Dias and F.J. Enguita, The circulating non-coding RNA landscape for biomarker research: lessons and prospects from cardiovascular diseases. Acta Pharmacol Sin, 2018. 39(7): p. 1085-1099.

22. Komal, S., J.J. Yin, S.H. Wang, C.Z. Huang, H.L. Tao, J.Z. Dong, et al., MicroRNAs: Emerging biomarkers for atrial fibrillation. J Cardiol, 2019.

23. Platonov, P.G., L.B. Mitrofanova, V. Orshanskaya and S.Y. Ho, Structural abnormalities in atrial walls are associated with presence and persistency of atrial fibrillation but not with age. J Am Coll Cardiol, 2011. 58(21): p. 2225-2232.

24. Yamaguchi, T., T. Tsuchiya, A. Fukui, Y. Kawano, T. Otsubo, Y. Takahashi, et al., Impact of the extent of low-voltage zone on outcomes after voltage-based catheter ablation for persistent atrial fibrillation. J Cardiol, 2018. 72(5): p. 427-433.

25. Mohanty, S., P. Mohanty, L. Di Biase, C. Trivedi, E.H. Morris, C. Gianni, et al., Long-term follow-up of patients with paroxysmal atrial fibrillation and severe left atrial scarring: comparison between pulmonary vein antrum isolation only or pulmonary vein isolation combined with either scar homogenization or trigger ablation. Europace, 2017. 19(11): p. 1790-1797.

26. Kholmovski, E.G., A.K. Morris and M.G. Chelu, Cardiac MRI and Fibrosis Quantification. Card Electrophysiol Clin, 2019. 11(3): p. 537-549.

27. Nattel, S., How does fibrosis promote atrial fibrillation persistence:in silicofindings, clinical observations, and experimental data. Cardiovascular Research, 2016. 110(3): p. 295-297.

28. Landstrom, A.P., D. Dobrev and X.H.T. Wehrens, Calcium Signaling and Cardiac Arrhythmias. Circ Res, 2017. 120(12): p. 1969-1993.

29. Calvo, D., D. Filgueiras-Rama and J. Jalife, Mechanisms and Drug Development in Atrial Fibrillation. Pharmacol Rev, 2018. 70(3): p. 505-525.

30. Morishima, M., E. Iwata, C. Nakada, Y. Tsukamoto, H. Takanari, S. Miyamoto, et al., Atrial FibrillationMediated Upregulation of miR-30d Regulates Myocardial Electrical Remodeling of the G-ProteinGated K(+) Channel, IK.ACh. Circ J, 2016. 80(6): p. 1346-1355.

31. Shan, H., Y. Zhang, Y. Lu, Y. Zhang, Z. Pan, B. Cai, et al., Downregulation of miR-133 and miR-590 contributes to nicotine-induced atrial remodelling in canines. Cardiovasc Res, 2009. 83(3): p. 465472. 
32. Marrouche, N.F., D. Wilber, G. Hindricks, P. Jais, N. Akoum, F. Marchlinski, et al., Association of atrial tissue fibrosis identified by delayed enhancement MRI and atrial fibrillation catheter ablation: the DECAAF study. JAMA, 2014. 311(5): p. 498-506.

\section{Tables}

Table 1: characteristics of miRNAs differentially expressed between patients with AF and healthy controls

\begin{tabular}{|llllll|}
\hline Upregulated miRNAs & Fold Change & $P$ & Downregulated miRNAs & Fold Change & $P$ \\
\hline hsa-miR-302 & 5.43 & $<0.001$ & hsa-miR-199-3p & 0.16 & $<0.001$ \\
\hline hsa-miR-193a-5p & 14.74 & $<0.001$ & hsa-miR-1260 & 0.15 & $<0.001$ \\
hsa-miR-153-3p & 8.14 & $<0.001$ & hsa-miR-144-3p & 0.11 & $<0.001$ \\
hsa-miR-551-3p & 5.63 & $<0.001$ & hsa-miR-425-5p & 0.11 & $<0.001$ \\
& & & hsa-miR-21 & 0.15 & $<0.001$ \\
\hline & & hsa-miR-22-3p & 0.21 & $<0.001$ \\
\hline
\end{tabular}

\section{Figures}




\section{AF AF AF Ctl Ctl Ctl}

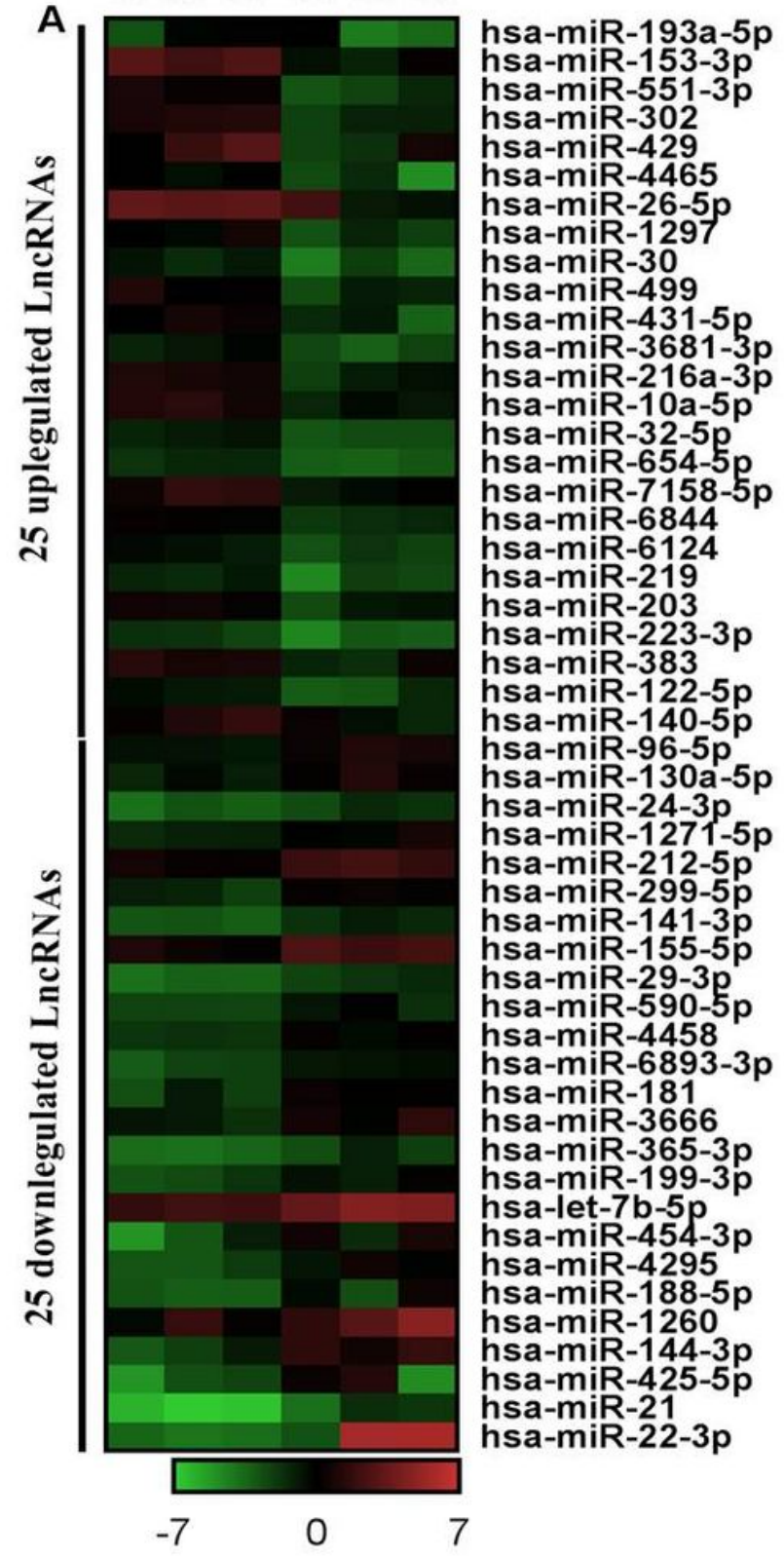

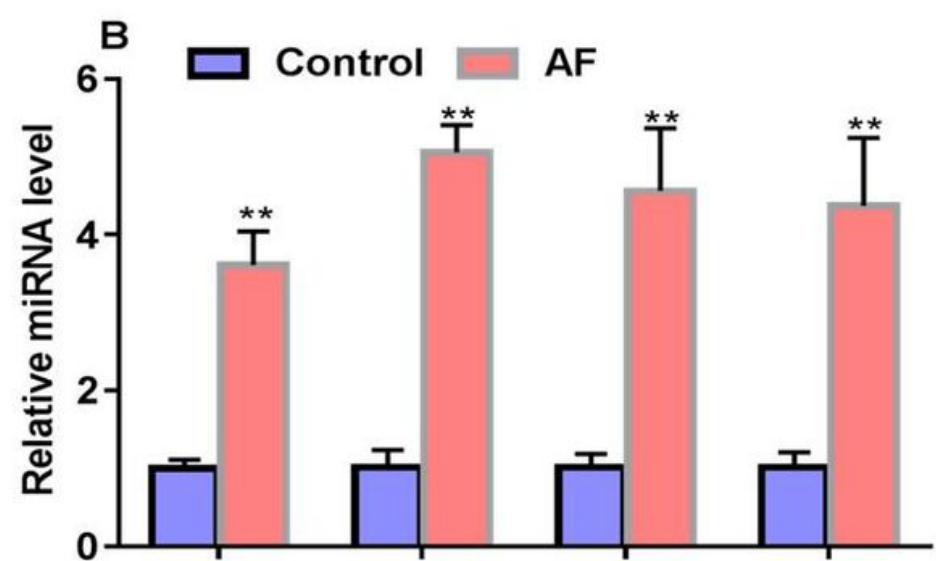
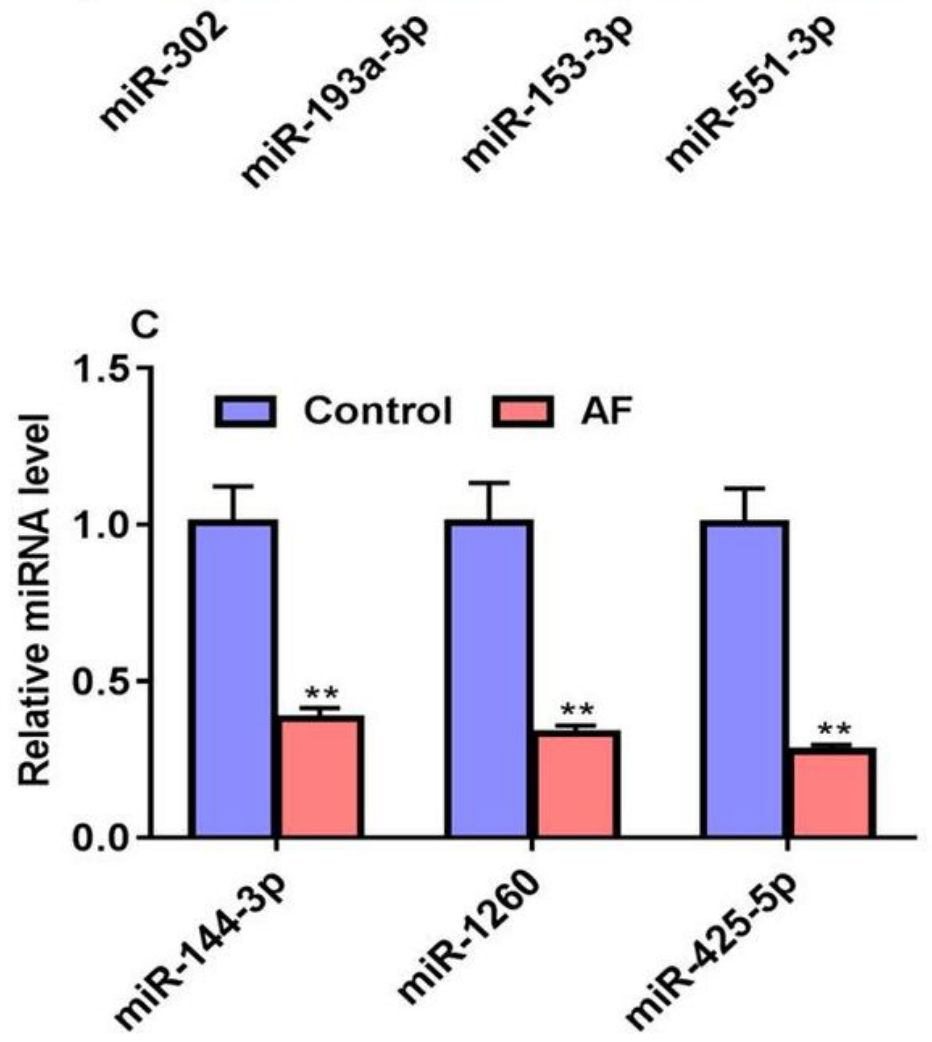

Figure 1

The expression level of miRNAs in plasma in $3 \mathrm{AF}$ patients and 3 healthy control by miRNA sequencing and qRT-PCR. A: Heatmap of miRNA expression data from plasma samples of patients with $A F(n=3)$ and healthy controls $(n=3)$. Relative miRNA expression is depicted according to the color scale shown on the botton. Red indicated upregulation; green indicated downregulation, $\mathrm{Ctl}$ indicated healthy control samples. B-C: Validation of miRNA sequencing data by qRT-PCR in patients with $\operatorname{AF}(n=3)$ and healthy controls $(n=3), * * P<0.01$ vs control. 

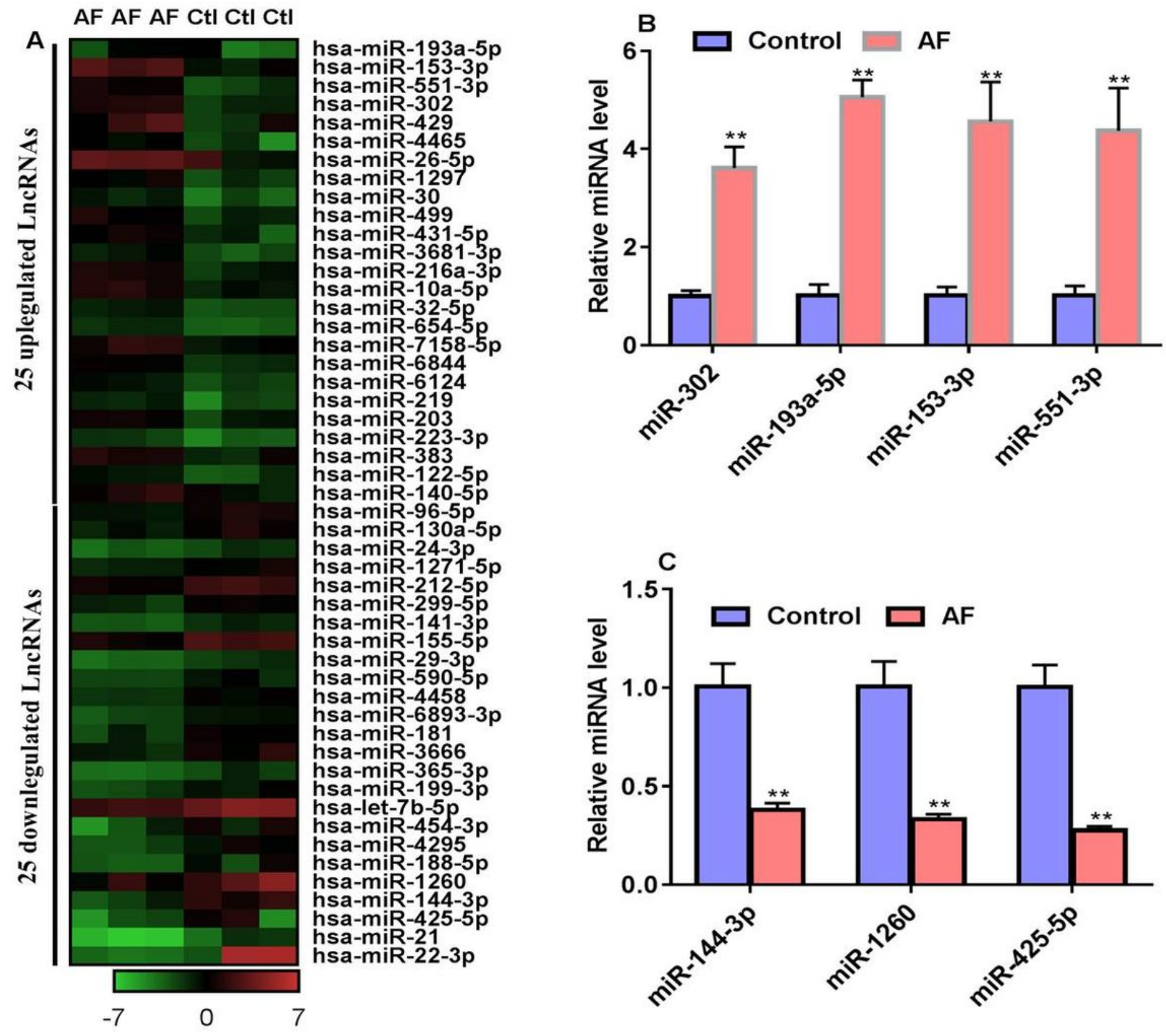

Figure 1

The expression level of miRNAs in plasma in $3 \mathrm{AF}$ patients and 3 healthy control by miRNA sequencing and qRT-PCR. A: Heatmap of miRNA expression data from plasma samples of patients with $A F(n=3)$ and healthy controls $(n=3)$. Relative miRNA expression is depicted according to the color scale shown on the botton. Red indicated upregulation; green indicated downregulation, Ctl indicated healthy control samples. B-C: Validation of miRNA sequencing data by qRT-PCR in patients with $A F(n=3)$ and healthy controls $(n=3), * \star P<0.01$ vs control. 

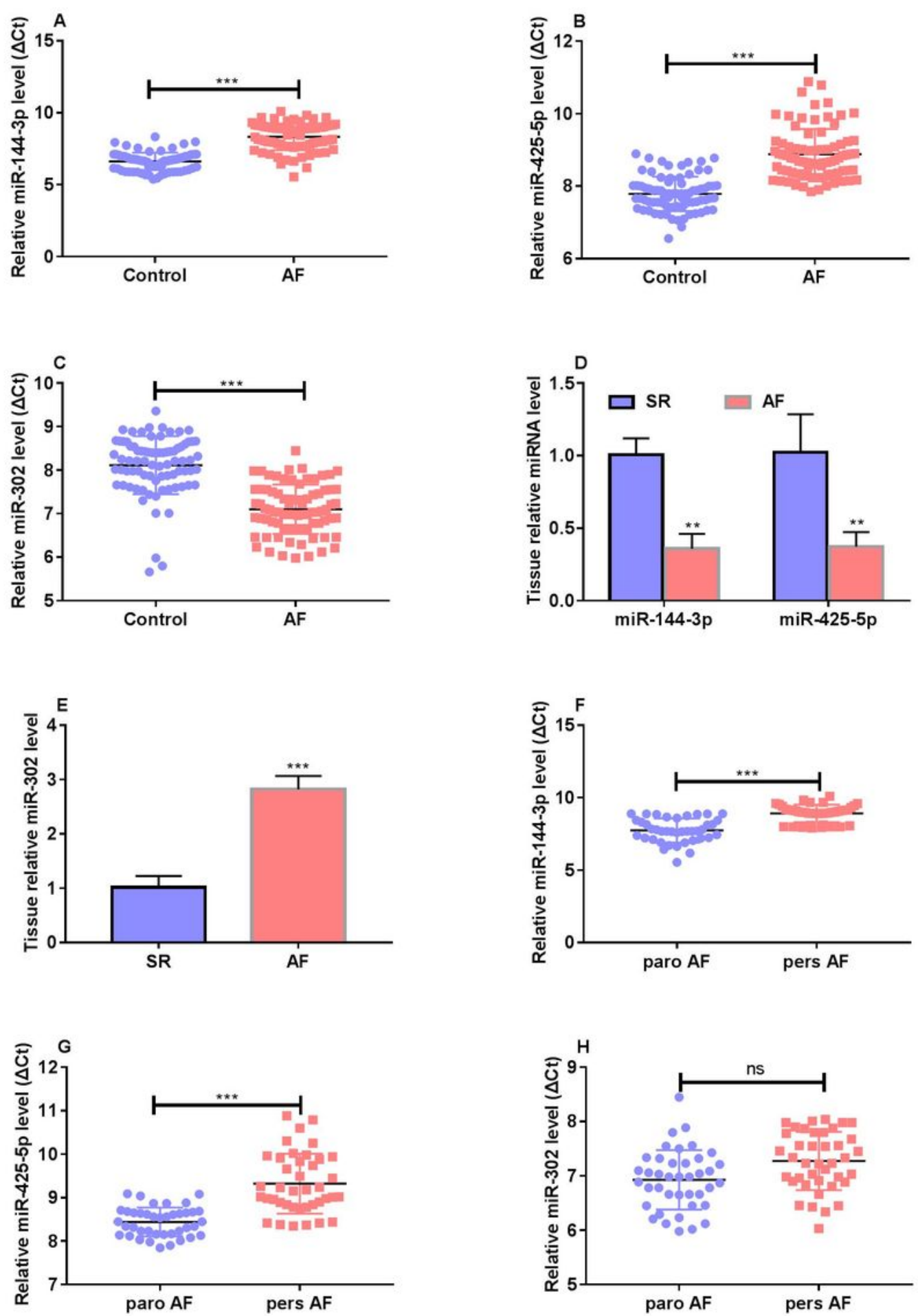

Figure 2

The difference expression miRNAs in an other two independent cohort and in different type AF. A-C: Validation of miRNA sequencing data by qRT-PCR in an independent cohort with $80 \mathrm{AF}$ patients and 80 healthy control, $\star \star \star *<00.001$. D-E: The expression level of miR-144-3p, miR-425-5p and miR-302 in atrial tissue in an independent cohort with $6 \mathrm{AF}$ and $6 \mathrm{SR}$ patients, ${ }^{\star \star *} \mathrm{P}<0.001,{ }^{\star *} \mathrm{P}<0.01$. F-H: The plasma level of miR-144-3p, miR-425-5p and miR-302 in 40 paroxysmal AF and 40 persistent $A F,{ }^{\star} * \star P<0.001$, paro: 
paroxysmal AF, pers: persistent AF, miR-16 was used as a reference gene for plasma, $\mathrm{U} 6$ was used as a reference gene for tissue. miRNA was calculated by the 2- $\Delta \Delta \mathrm{Ct}$ method or $\Delta \mathrm{Ct}, \Delta \mathrm{CtmiRNA}=\mathrm{Ct}$ targetmiRNA-Ct miR-16, and lower $\Delta C t$ value mean higher expression of miRNA.
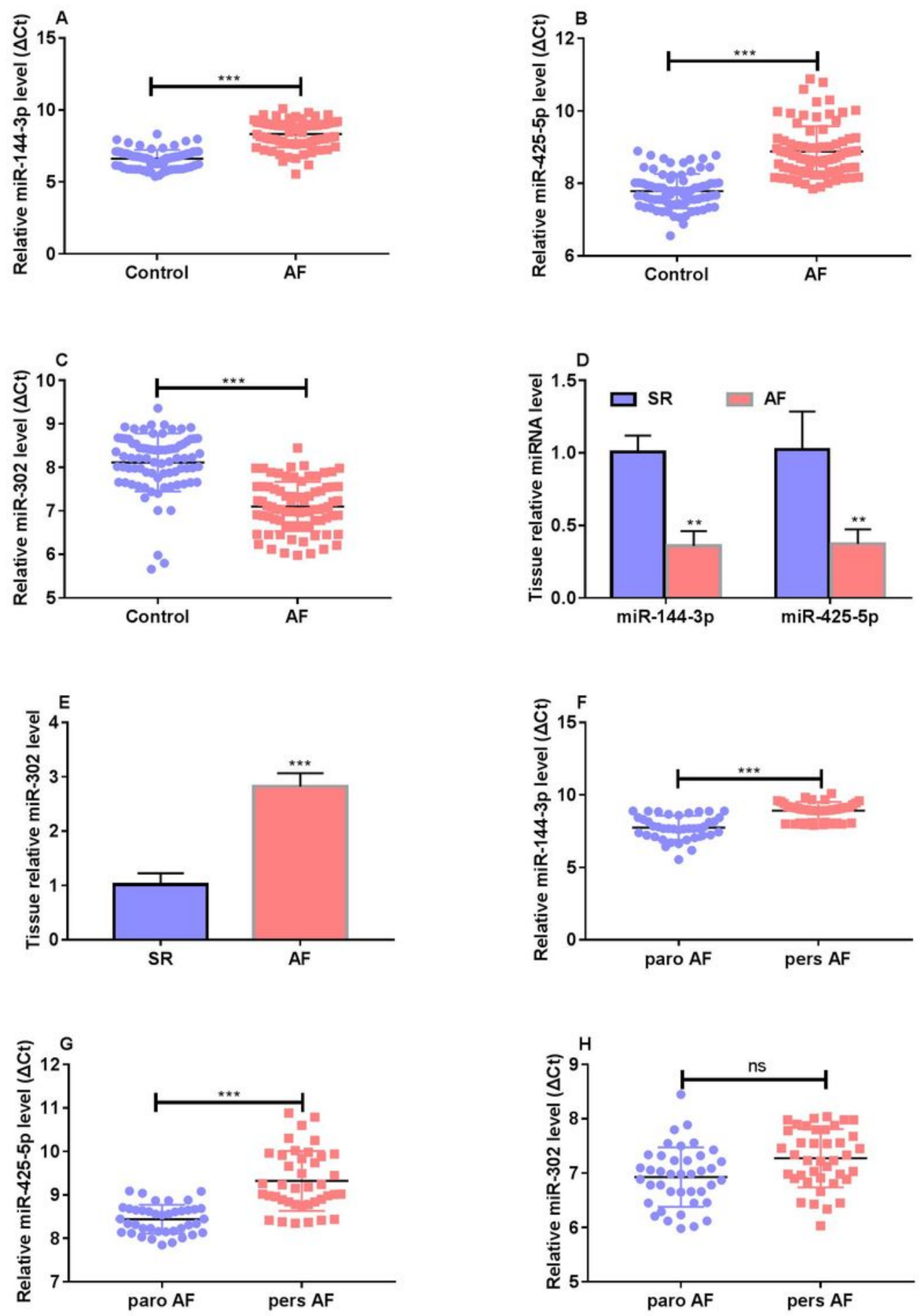

Figure 2

The difference expression miRNAs in an other two independent cohort and in different type AF. A-C: Validation of miRNA sequencing data by qRT-PCR in an independent cohort with $80 \mathrm{AF}$ patients and 80 
healthy control, ${ }^{\star} \star * P<0.001$. D-E: The expression level of miR-144-3p, miR-425-5p and miR-302 in atrial tissue in an independent cohort with $6 \mathrm{AF}$ and $6 \mathrm{SR}$ patients, ${ }^{\star \star *} \mathrm{P}<0.001,{ }^{\star \star} \mathrm{P}<0.01$. F-H: The plasma level of miR-144-3p, miR-425-5p and miR-302 in 40 paroxysmal AF and 40 persistent $A F, \star \star \star P<0.001$, paro: paroxysmal AF, pers: persistent AF, miR-16 was used as a reference gene for plasma, $\mathrm{U} 6$ was used as a reference gene for tissue. miRNA was calculated by the 2- $\Delta \Delta \mathrm{Ct}$ method or $\Delta \mathrm{Ct}, \Delta \mathrm{CtmiRNA}=\mathrm{Ct}$ targetmiRNA-Ct miR-16, and lower $\Delta \mathrm{Ct}$ value mean higher expression of miRNA.
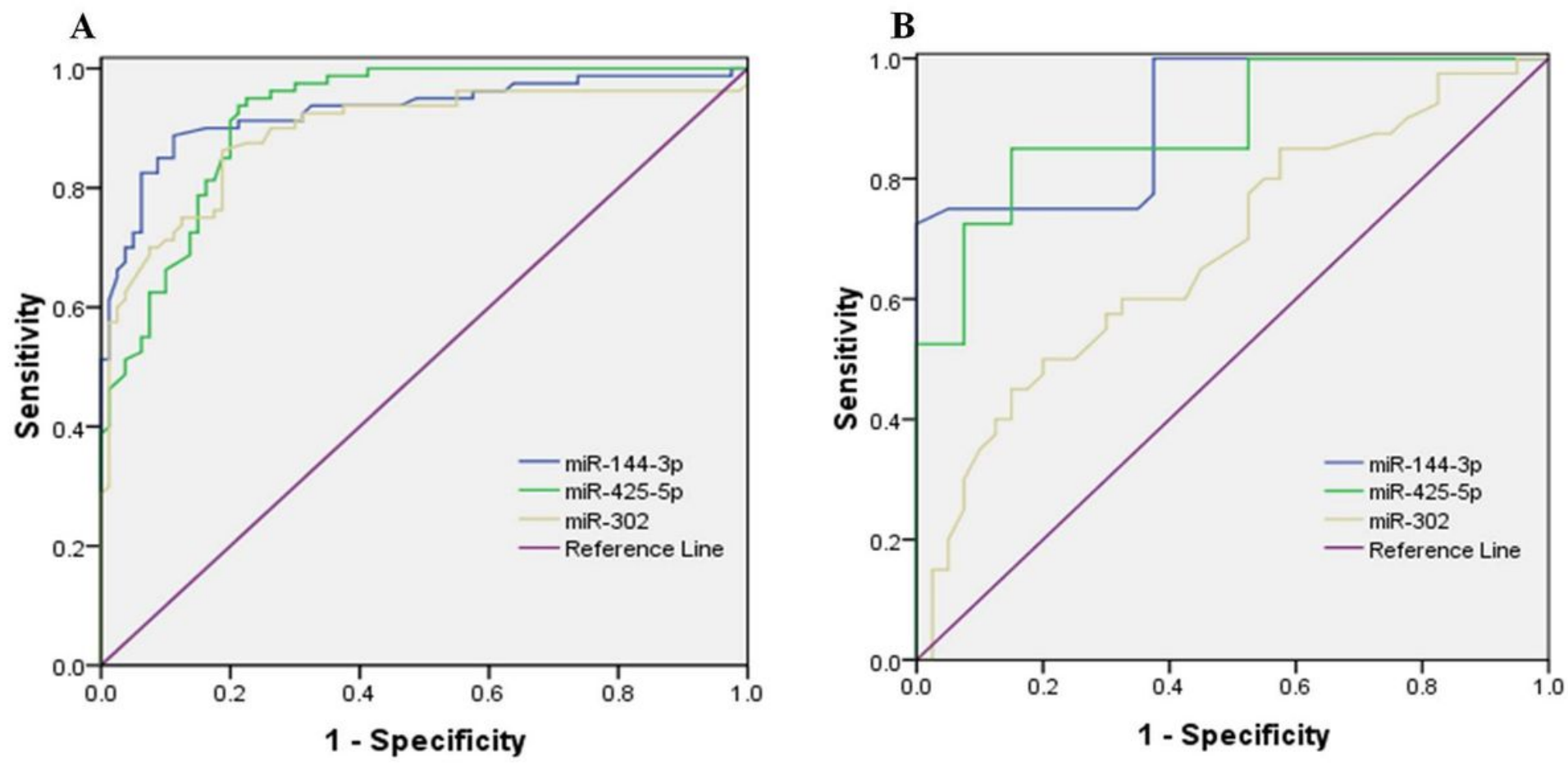

\begin{tabular}{|lcc|}
\hline Biomarkers & AUC & $95 \% \mathrm{CI}$ \\
miR-144-3p & 0.928 & $0.885-0.970$ \\
miR-425-5p & 0.921 & $0.881-0.961$ \\
miR-302 & 0.891 & $0.838-0.945$ \\
\hline
\end{tabular}

\begin{tabular}{|lcc|}
\hline Biomarkers & AUC & $95 \% \mathrm{CI}$ \\
miR-144-3p & 0.906 & $0.842-0.969$ \\
miR-425-5p & 0.888 & $0.817-0.958$ \\
miR-302 & 0.681 & $0.564-0.798$ \\
\hline
\end{tabular}

Figure 3

The diagnosis value of miRNAs for atrial fibrillation and the valve to differentiate persistent AF from paroxysmal AF by receiver operating characteristic (ROC) curve analysis. A. The diagnosis value of the miRNAs to discriminate AF from healthy control. B: The diagnosis value of the miRNAs to discriminate persistent $A F$ from paroxysmal $A F$. 
A

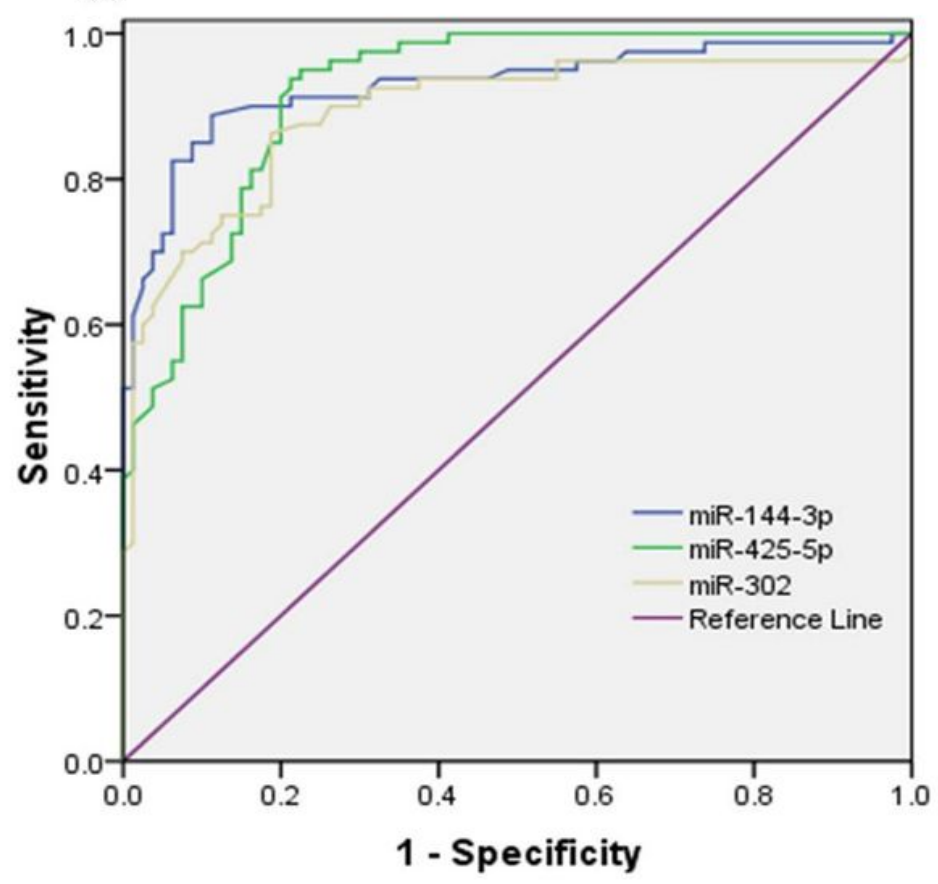

\begin{tabular}{|lcc|}
\hline Biomarkers & AUC & $95 \% \mathrm{CI}$ \\
miR-144-3p & 0.928 & $0.885-0.970$ \\
miR-425-5p & 0.921 & $0.881-0.961$ \\
miR-302 & 0.891 & $0.838-0.945$ \\
\hline
\end{tabular}

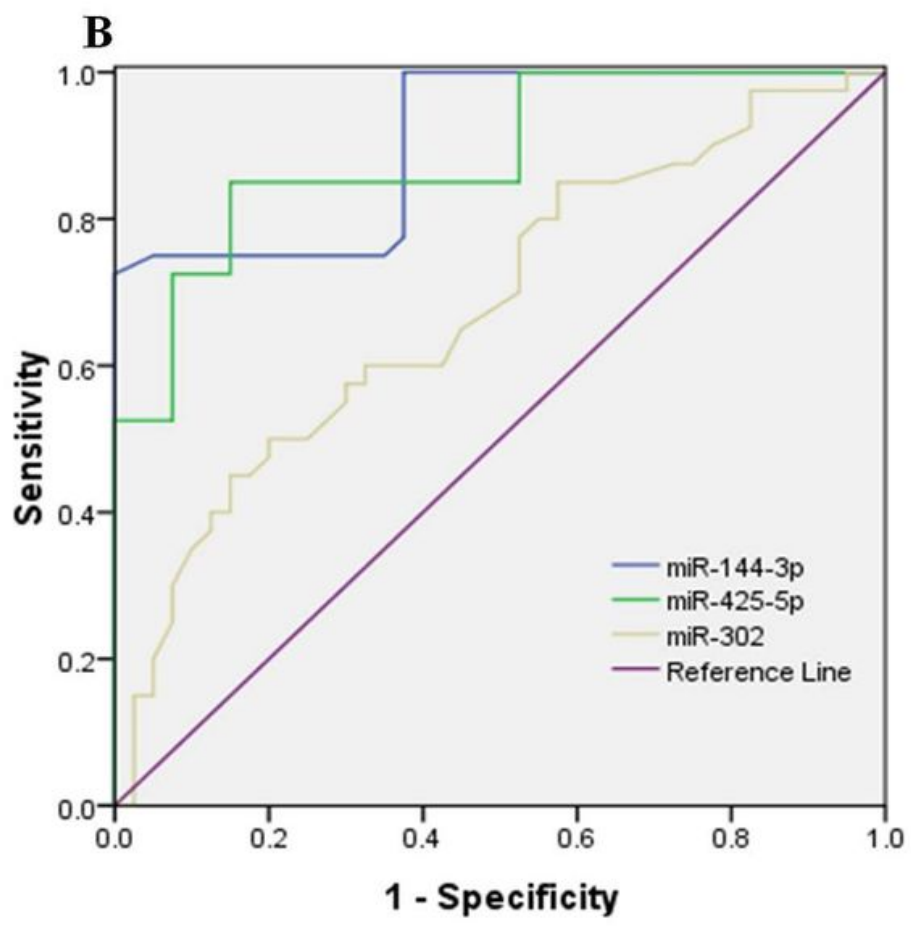

\begin{tabular}{|lcc|}
\hline Biomarkers & AUC & $95 \% \mathrm{CI}$ \\
miR-144-3p & 0.906 & $0.842-0.969$ \\
miR-425-5p & 0.888 & $0.817-0.958$ \\
miR-302 & 0.681 & $0.564-0.798$ \\
\hline
\end{tabular}

\section{Figure 3}

The diagnosis value of miRNAs for atrial fibrillation and the valve to differentiate persistent AF from paroxysmal AF by receiver operating characteristic (ROC) curve analysis. A. The diagnosis value of the miRNAs to discriminate AF from healthy control. B: The diagnosis value of the miRNAs to discriminate persistent AF from paroxysmal AF. 

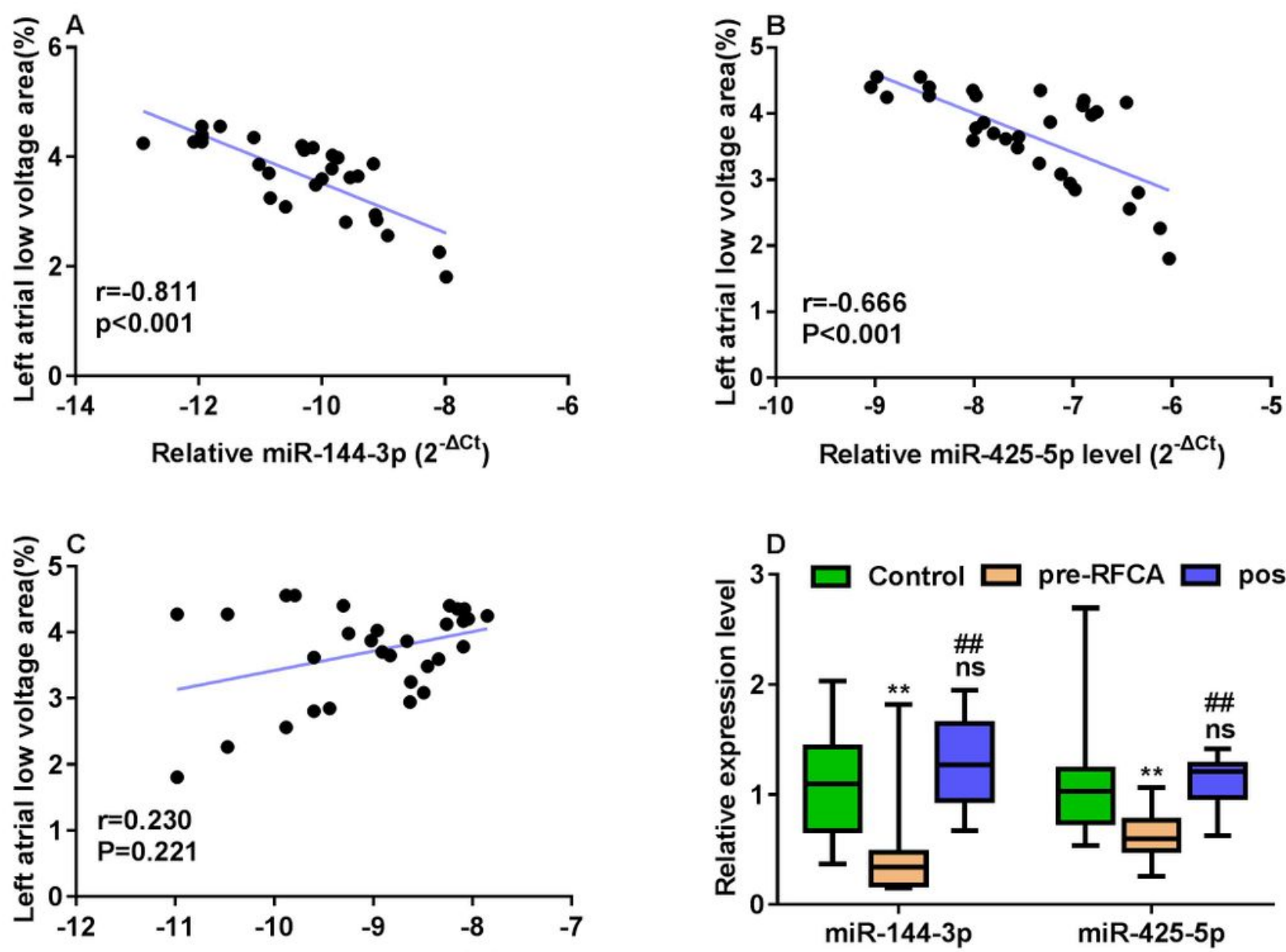

Relative miR-302 level $\left(2^{-\Delta C t}\right)$
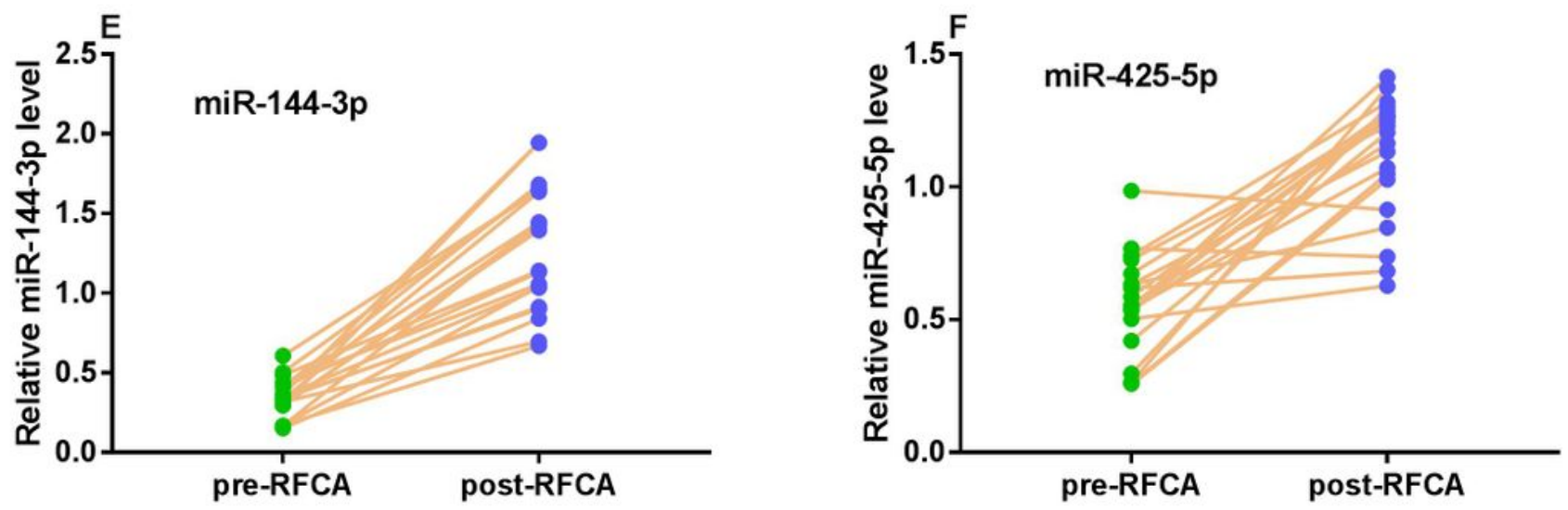

Figure 4

The correlation between miRNAs and left atrial fibrosis, and the difference expression of miR-144-3p and miR-425-5p in pre-RFCA and post-RFCA in plasma. A: The correlation between plasma miR-144-3p and the extent of left atrial low voltage area. B: The correlations between plasma miR-425-5p and the extent of left atrial low voltage area. C: The correlations between plasma miR-302 and the extent of left atrial low voltage area, all valve were log-transformed to normalize their distribution before statistical analysis, 
the extent of left atrial low voltage was used to evaluate left fibrosis. D: The plasma level of miR-144-3p and miR-425-5p in the control group $(n=20)$ and pre-RFCA $(n=20)$, post-RFCA $(n=20),{ }^{\star} P<0.01$ vs control group, \#\#P<0.01 vs pre-RFCA, ns: no significant vs Control group. E: The expression change of miR-144$3 p$ in each AF patients in pre-RFCA and post-RFCA. $F$ : The expression change of miR-425-5p in each AF patients in pre-RFCA and post-RFCA.
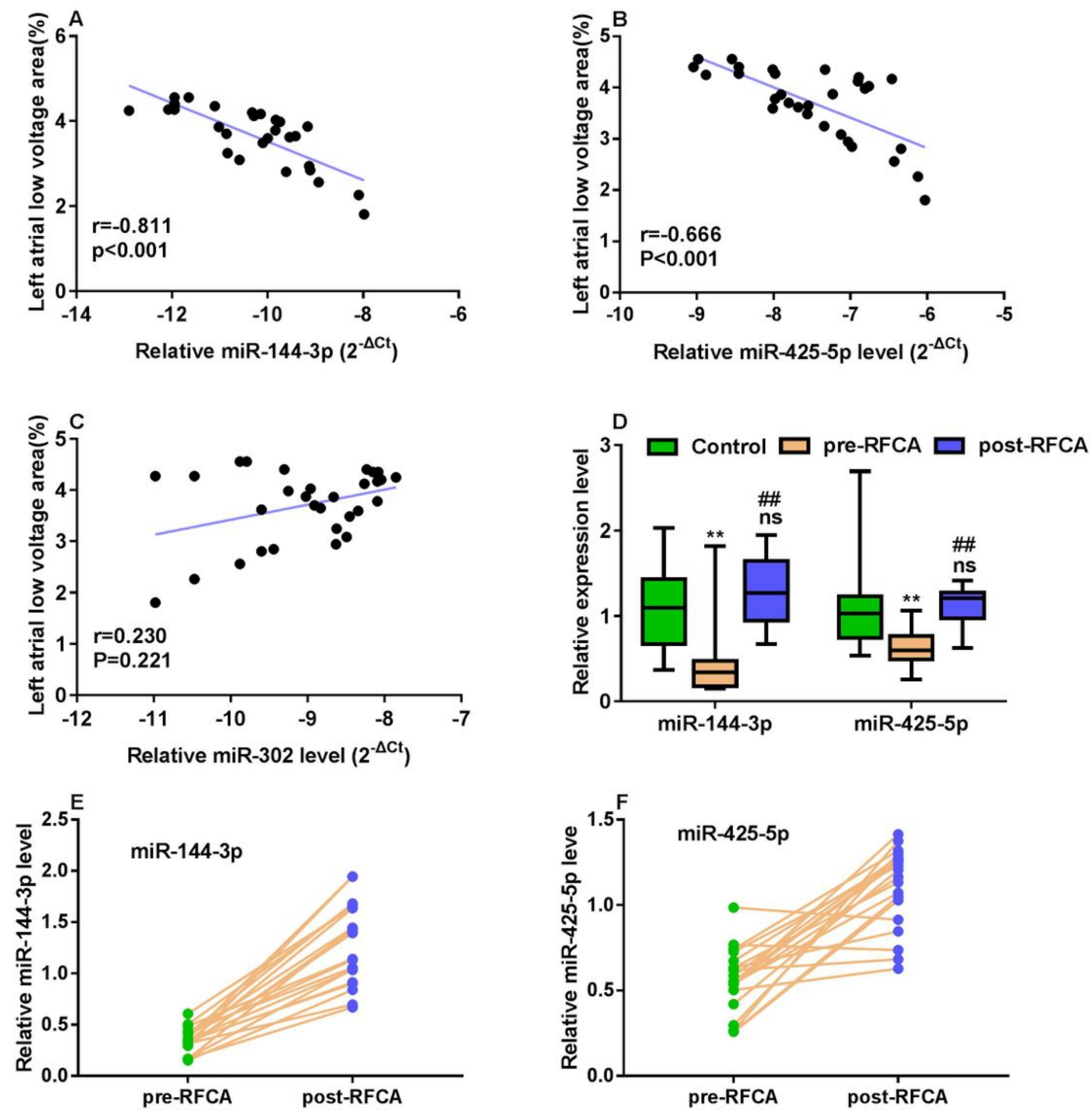

Figure 4 
The correlation between miRNAs and left atrial fibrosis, and the difference expression of miR-144-3p and miR-425-5p in pre-RFCA and post-RFCA in plasma. A: The correlation between plasma miR-144-3p and the extent of left atrial low voltage area. B: The correlations between plasma miR-425-5p and the extent of left atrial low voltage area. C: The correlations between plasma miR-302 and the extent of left atrial low voltage area, all valve were log-transformed to normalize their distribution before statistical analysis, the extent of left atrial low voltage was used to evaluate left fibrosis. D: The plasma level of miR-144-3p and miR-425-5p in the control group $(n=20)$ and pre-RFCA $(n=20)$, post-RFCA $(n=20),{ }^{\star} P<0.01$ vs control group, \#\#P<0.01 vs pre-RFCA, ns: no significant vs Control group. $E$ : The expression change of miR-144$3 p$ in each AF patients in pre-RFCA and post-RFCA. $F$ : The expression change of miR-425-5p in each AF patients in pre-RFCA and post-RFCA. 

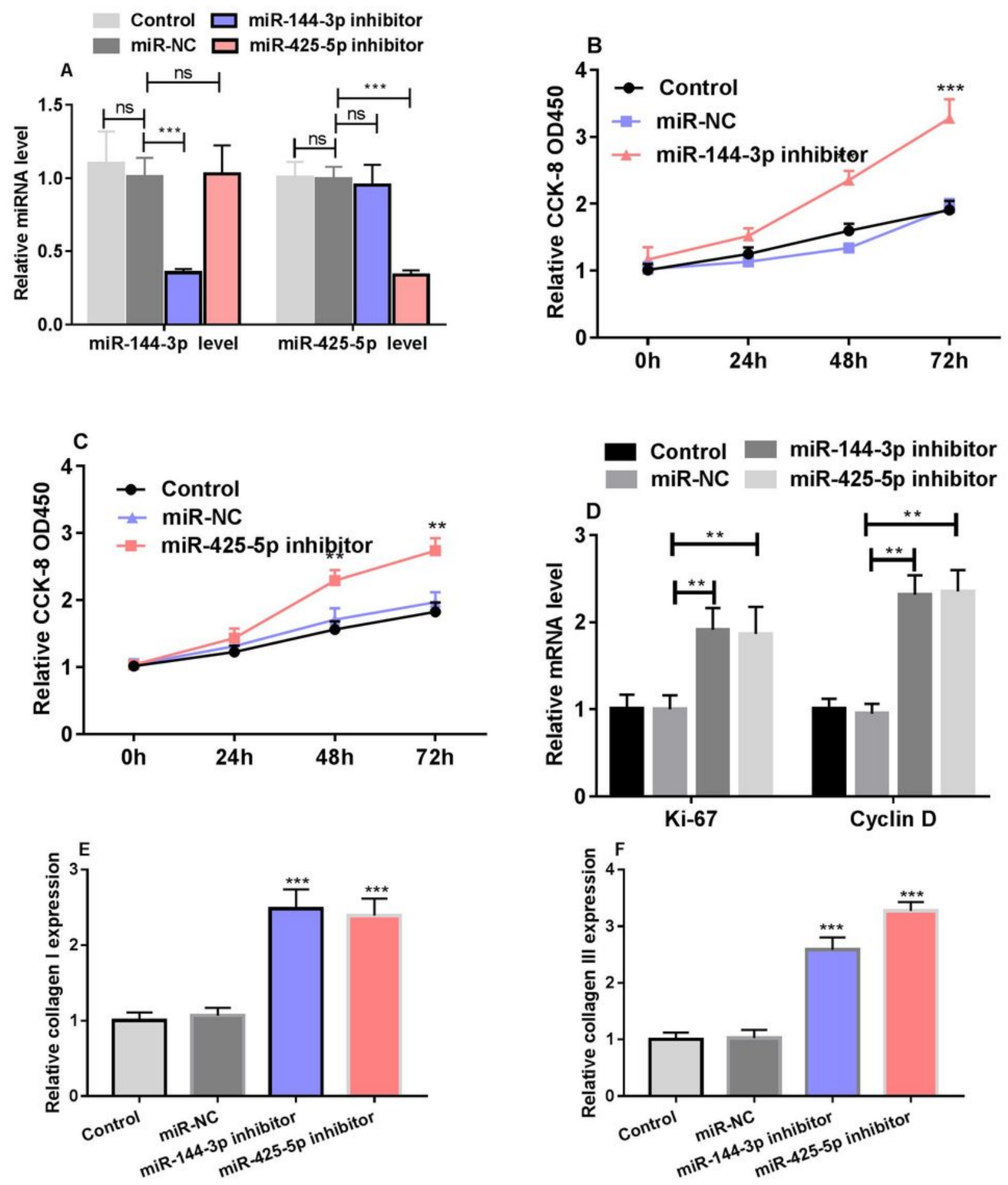

Figure 5

Effect of miR-144-3p and miR-425-5p on fibroblast cell proliferation and fibrosis. A: miR-144-3p and miR425-5p expression level in fibroblast cell transfected with miR-144-3p or miR-425-5p inhibitor. B-C:

Fibroblast cell proliferation was detected with CCK-8. D: The proliferation markers of Ki67 and Cyclin D. E$F$ : The expression level of fibrosis biomarkers of $A F, * * * P<0.001, * * P<0.01$. 

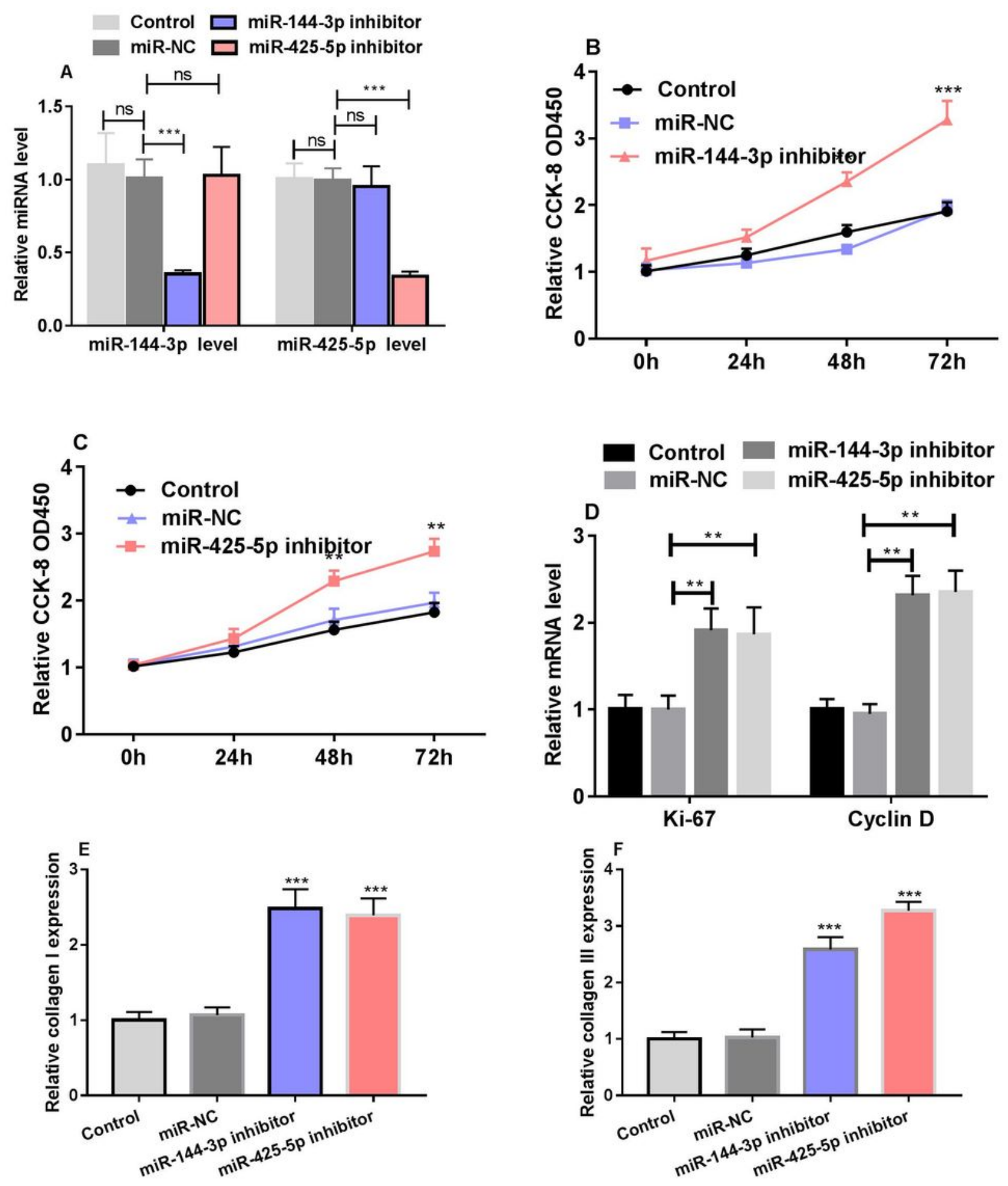

Figure 5

Effect of miR-144-3p and miR-425-5p on fibroblast cell proliferation and fibrosis. A: miR-144-3p and miR425-5p expression level in fibroblast cell transfected with miR-144-3p or miR-425-5p inhibitor. B-C:

Fibroblast cell proliferation was detected with CCK-8. D: The proliferation markers of Ki67 and Cyclin D. EF: The expression level of fibrosis biomarkers of $A F,{ }^{\star *}{ }^{2} P<0.001, * * P<0.01$. 

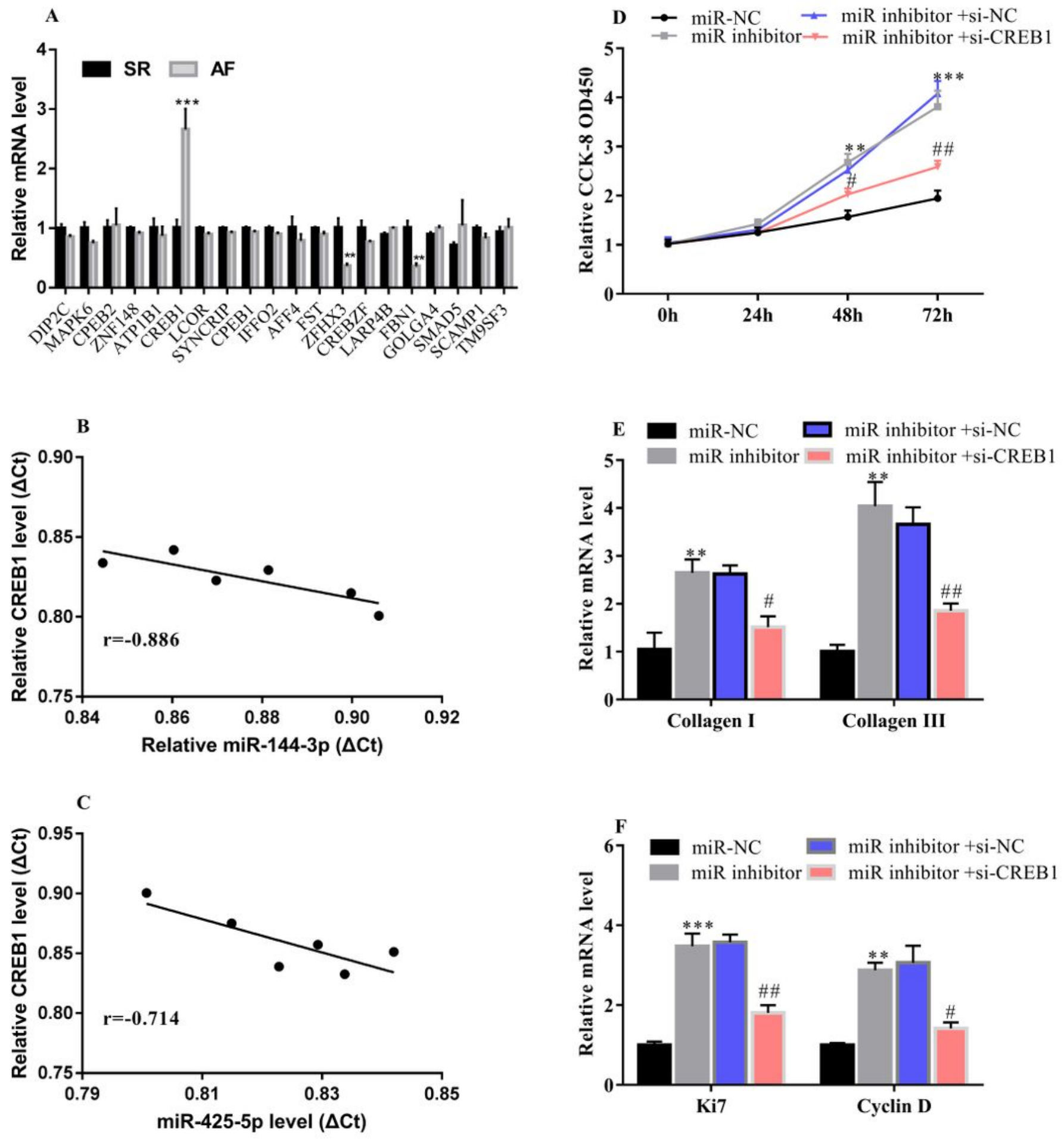

Figure 6

CREB1 was the common target gene of miR-144-3p and miR-425-5p and inhibition of CREB1 expression could reverse the phenotype of down-regulation of miR-144-3p and miR-425-5p. A: CREB1 was upregulated in atrial tissue, but ZFHX3 and FBN1 was downregulated of the 20 common target genes in atrial tissue in AF patients, ${ }^{\star *} \mathrm{P}<0.01$ vs SR. B-C: miR-144-3p and miR-425-5p was negatively associated with CREB1 by Pearson's correlation coefficients. D: Fibroblast cell proliferation was detected with CCK-8. 
E: The proliferation markers of Ki67 and Cyclin. F: The expression level of fibrosis biomarkers of AF, ${ }^{\star} \star * \mathrm{P}<0.001,{ }^{\star *} \mathrm{P}<0.01$ vs miR-NC. \#\#P<0.01, \#P<0.05 vs miR inhibitor + si-NC.
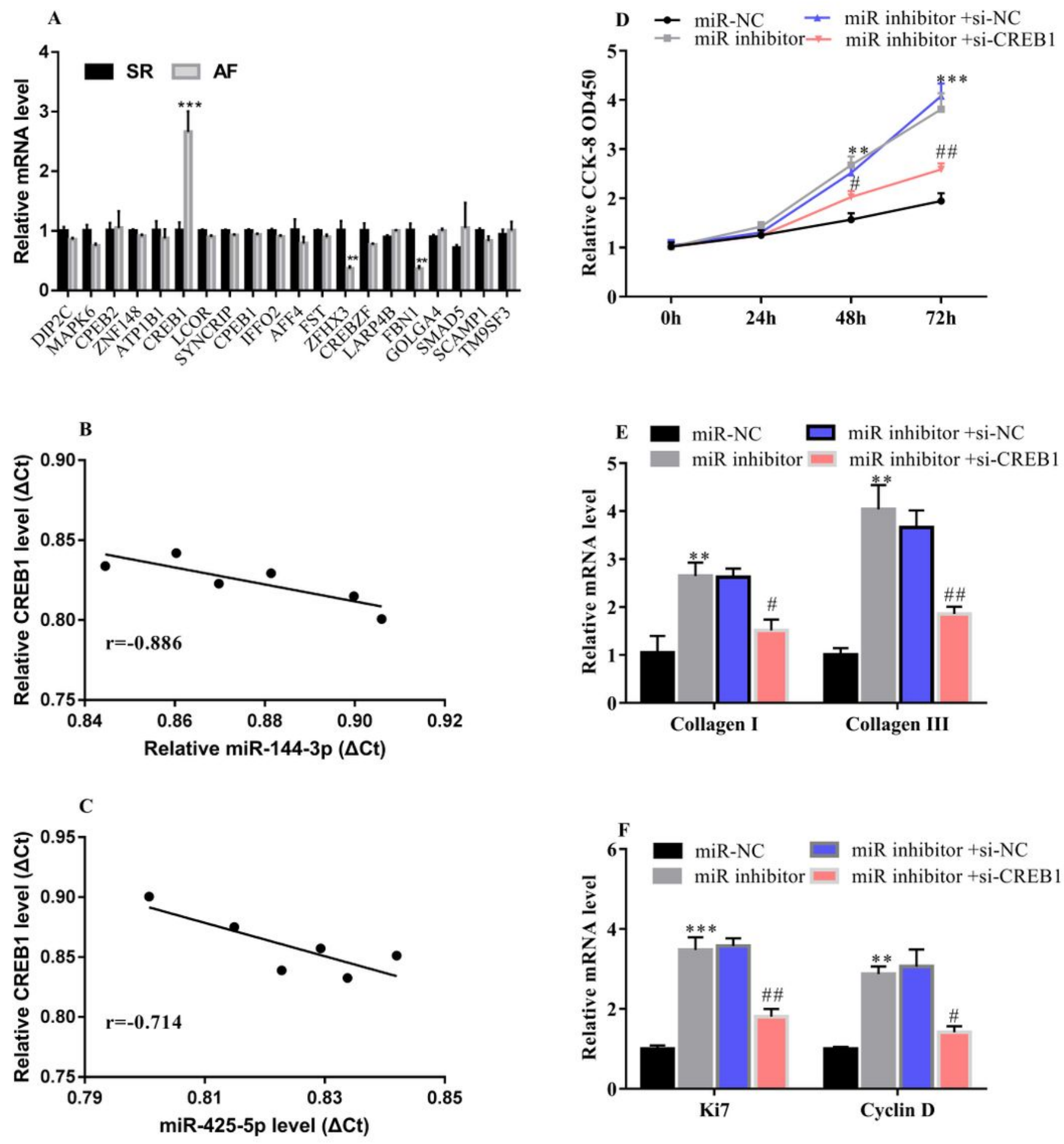

Figure 6

CREB1 was the common target gene of miR-144-3p and miR-425-5p and inhibition of CREB1 expression could reverse the phenotype of down-regulation of miR-144-3p and miR-425-5p. A: CREB1 was upregulated in atrial tissue, but ZFHX3 and FBN1 was downregulated of the 20 common target genes in 
atrial tissue in AF patients, ${ }^{\star *} \mathrm{P}<0.01$ vs SR. B-C: miR-144-3p and miR-425-5p was negatively associated with CREB1 by Pearson's correlation coefficients. D: Fibroblast cell proliferation was detected with CCK-8. E: The proliferation markers of Ki67 and Cyclin. F: The expression level of fibrosis biomarkers of AF, ${ }^{*} * * \mathrm{P}<0.001,{ }^{* *} \mathrm{P}<0.01$ vs miR-NC. \#\#P<0.01, \#P<0.05 vs miR inhibitor +si-NC.

A

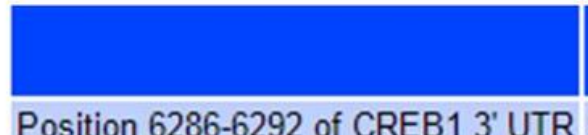

Position 6286-6292 of CREB1 3' UTR 5'

hsa-miR-144-3p

Position 6266-6272 of CREB1 3' UTR 5'

hsa-miR-425-5p

Position 6310-6316 of CREB1 3' UTR 5'

hsa-miR-425-5p

$$
\text { B }
$$

CREB1

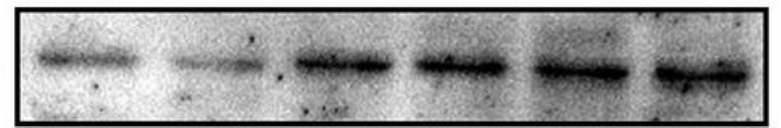

GAPDH

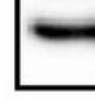

miR-NC

D

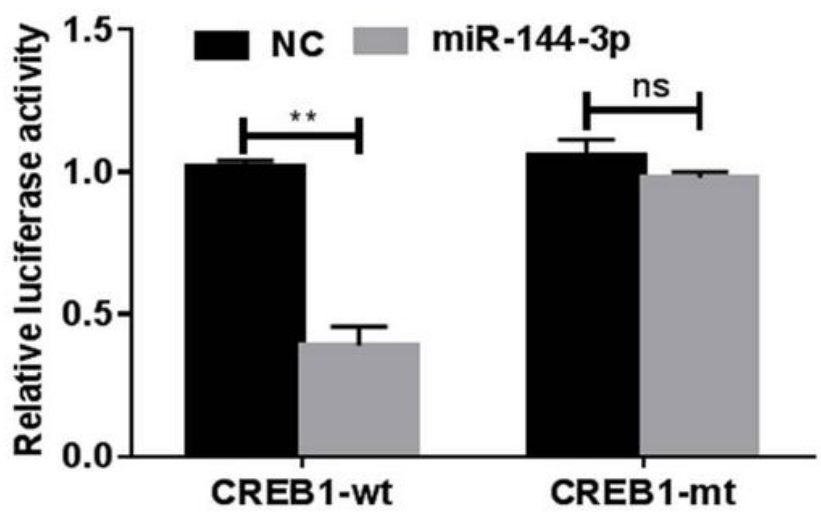

Predicted consequential pairing of target region (top) and miRNA (bottom) . . . UARAGUGCUUUUUUCUUACUGUAR. . .

UCAUGUAGUAGAUAUGACAU

IIIIII

$|I I I| \mid$

IIII

AGUUGCCCUCACUAGC--ACAGUAA

. . CUUUUGUGGUAUCAACUGUCAUAA. . .

AGUUGCCCUCACUAGCACAGUAA
Site type 7merA1

C
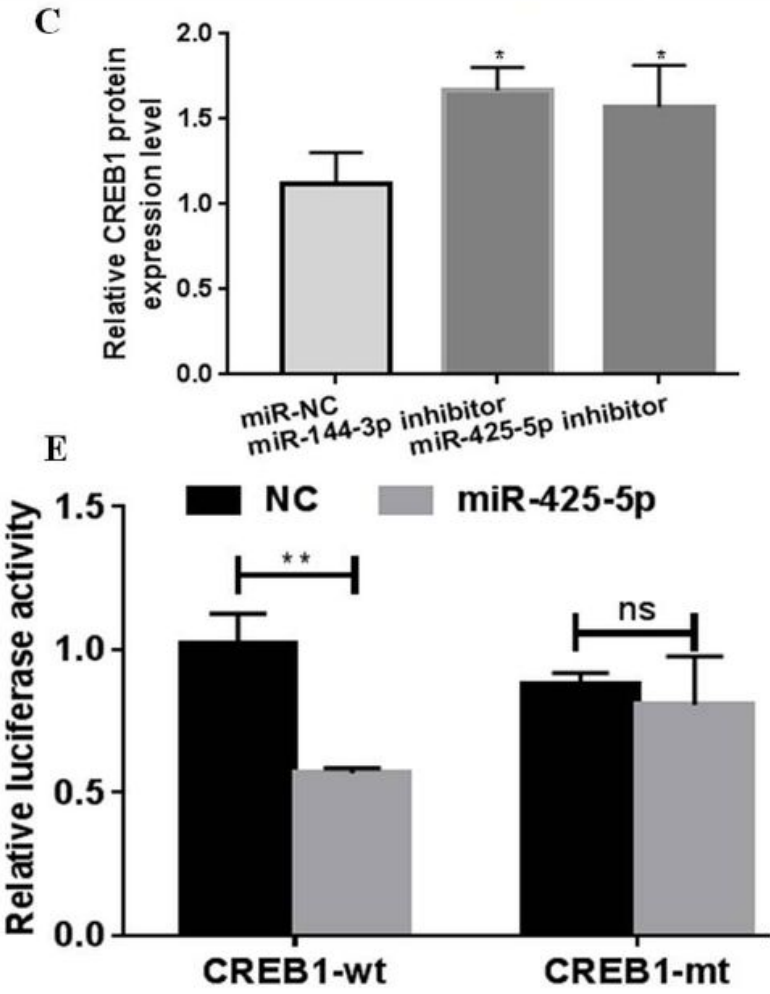

Figure 7

CREB1 was the cmmon direct target gene of miR-144-3p and miR-425-5p. A: The potential binding site of miR-144-3p and miR-425-5p with CREB1 3'-URT was indicated with TargetScan 7.2 database. B-C: The CREB1 protein expression level in atrial fibroblast cell transfected with miR-NC, miR-144-3p and miR-425$5 p$ inhibitor, GAPDH was used as loading control. D: The relative luciferase expression with CREB1 3'-UTR after co-transfection with miR-144-3p, miR-425-5p mimic or NC in HL-1 cell, ** $P<0.01,{ }^{*}<0.05$. 
Position 6286-6292 of CREB1 3' UTR 5' ... URARGUGCUUUUUUCUUACUGUAR. . . hsa-miR-144-3p $3^{\prime}$

UCAUGUAGUAGAUAUGACAU

IIIIII

IIIIII AGUUGCCCUCACUAGC--ACAGUAS hsa-miR-425-5p

Position 6310-6316 of CREB1 $3^{\prime}$ UTR $5^{\prime}$ hsa-miR-425-5p

B

CREB1

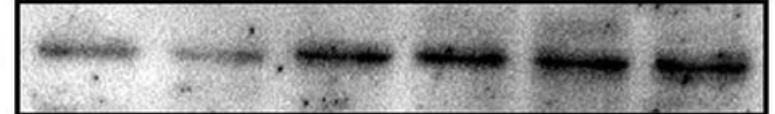

GAPDH

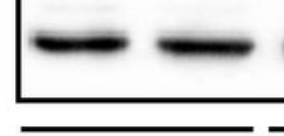

miR-NC

D

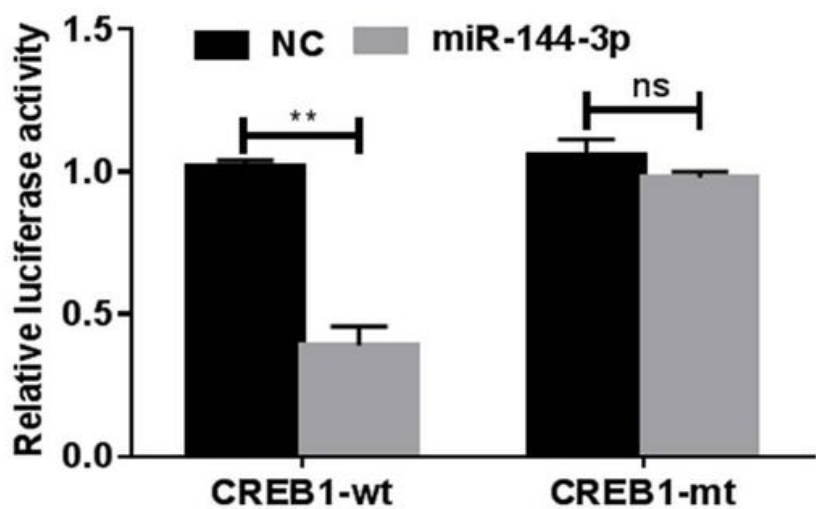
AGUUGCCCUCACUAGCACAGUAA

... CUUUGUGGUAUCAACUGUCAUAA. . .

| | | | |

A1

$-0.03$

C

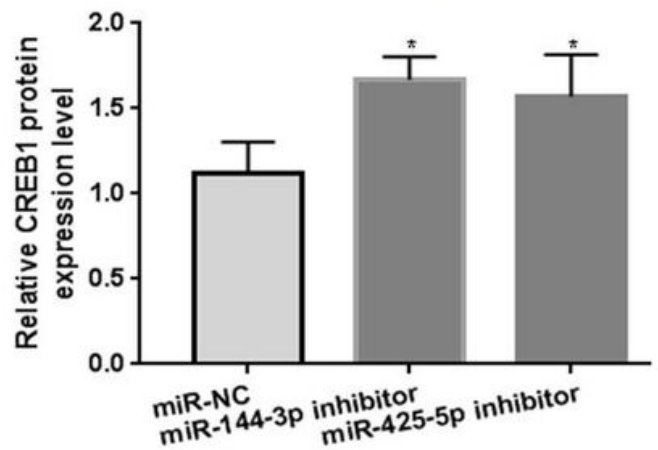

E

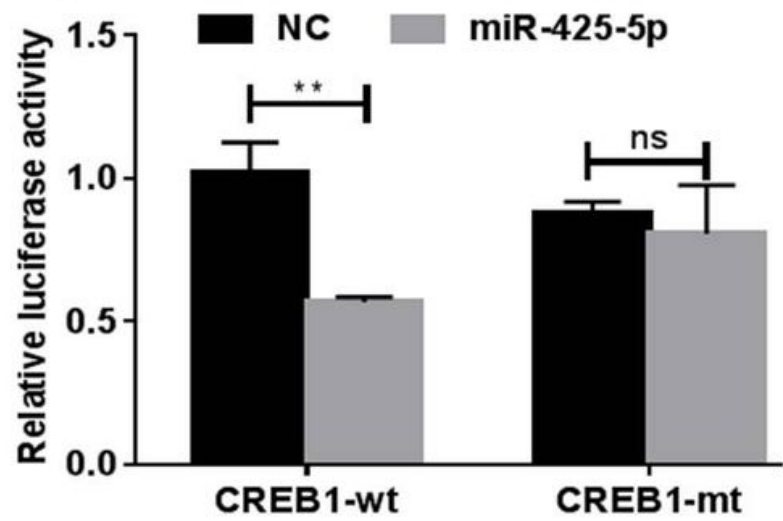

Figure 7

CREB1 was the cmmon direct target gene of miR-144-3p and miR-425-5p. A: The potential binding site of miR-144-3p and miR-425-5p with CREB1 3'-URT was indicated with TargetScan 7.2 database. B-C: The CREB1 protein expression level in atrial fibroblast cell transfected with miR-NC, miR-144-3p and miR-425$5 p$ inhibitor, GAPDH was used as loading control. D: The relative luciferase expression with CREB1 3'-UTR after co-transfection with miR-144-3p, miR-425-5p mimic or NC in HL-1 cell, **P<0.01, *P<0.05.

\section{Supplementary Files}

This is a list of supplementary files associated with this preprint. Click to download.

- Supplementdata.docx

- Supplementdata.docx 